然1118995(2)

PPPL-2629

UC-420
PREPARED FOR THE U.S. DEPARTMENT OF ENERGY, UNDER CONTRACT DE-AC02-76-CHO-3073

PPPL-2629

IGNITION PROBABILITIES FOR COMPACT IGNITION TOKAMAK DESIGNS

BY

D.P. STOTLER, R.J. GOLDSTON, AND THE CIT TEAM

September 1989
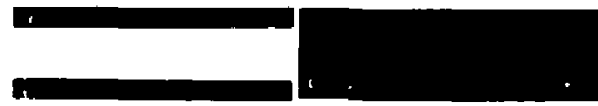

PRINCETON

phama phygice

LADOAATOAY

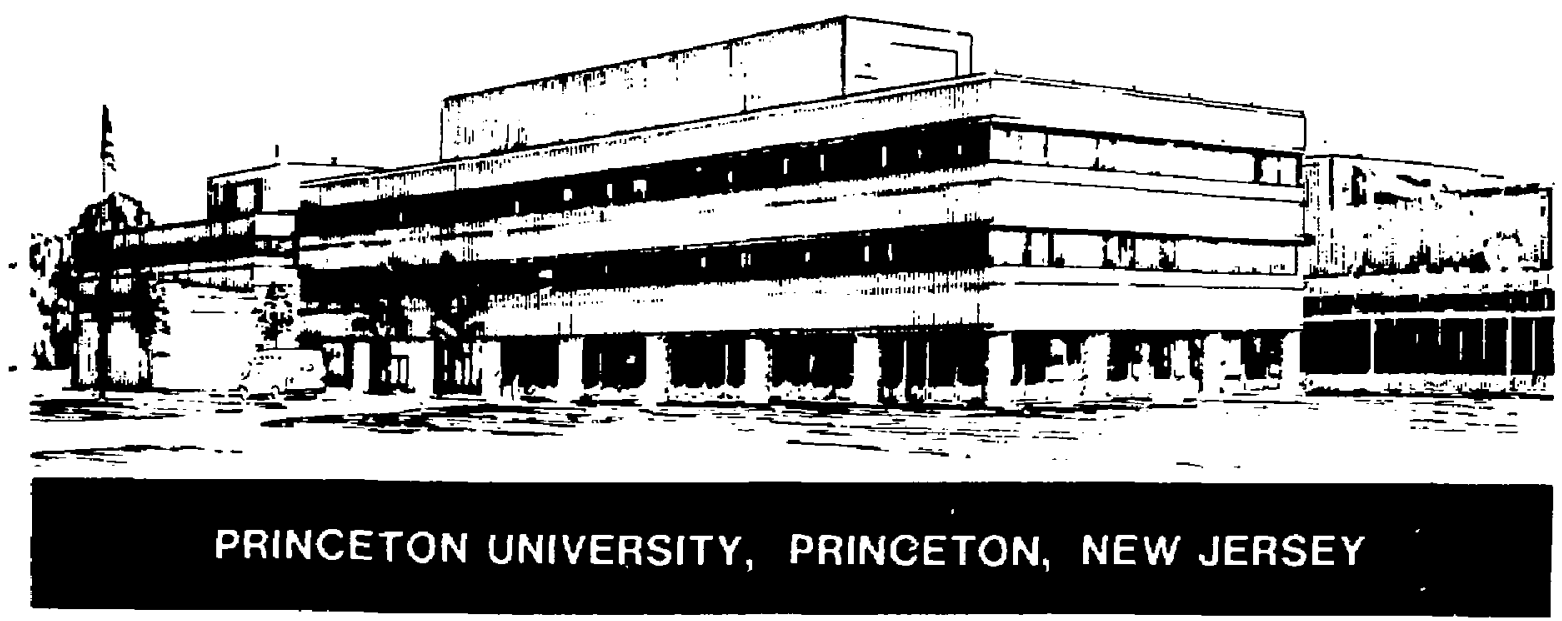

DISTRIBUTION OF THIS DCCDUVENT IS LINSMMTED 


\section{NOTICE}

Available from:

National Technical Information Service

U.S. Department of Commerce

5285 Port Royal Road

Springfield, Virginia 22161

$703-487-4650$

Use the following price codes when ordering:

Price: Printed Copy A03

Microfiche A01

\section{DISÉLAIMER}

\footnotetext{
This report was prepared as an account of work spunsored by an agency of the United States Government Netther lhe liniled Siates Guvernment nur any agency thereuf. nor any of their employecs. makes atsy warranly. express of implied ut assumes any legal hability of fesponsihilliy for the accutasy. cumpleteness, or uselulness of any informatuon, apparalus, produtt, or

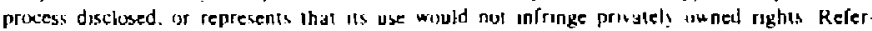
ence hesein io any specific commescial product. process. wr service by irade name, Iralemark, manufacturer, or otherwise dies nut neceisarily constllute ur imply its endirsement. reciom

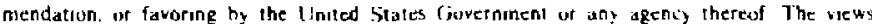
and opinuons of authors expressed hefein du nul necessarily sate ur reflect thuse of the 1 !nted States Goverament of any agency theresf
} 
PPPL- -2629

SEB9 016932

\title{
Ignition Probabilities for Compact Ignition Tokamak Designs
}

\author{
D. P. Stotler, R. J. Goldston, and the CIT Team \\ Princeton Plasma Physics Laboratory \\ Princeton University \\ Princeton, New Jersey 08543
}

\begin{abstract}
A global power balance code employing Monte Carlo techniques has been developed to study the "probability of ignition" [Nuci. Fusion, 29, 81 (1989)] and has been applied to several different configurations of the Compact Ignition Tokamak (CIT). Probability distributions for the critical physics parameters in the code were estimated using existing experimental data. This included a statistical evaluation of the uncertainty in extrapolating the energy confinement time. A substantial probability of ignition is predicted for CIT if peaked density profiles can be achieved or if one of the two higher plasma current configurations is employed. In other cases, values of the energy multiplication factor $Q$ of order 10 are generally obtained. The Ignitor-U and ARIES designs are also examined briefly. Comparisons of our empirically based confinement assumptions with two theory-based transport models yield conflicting results.
\end{abstract}




\section{Introduction}

Over the past several years, simple, global power balance calculations have been used to predict performance of reactor designs. ${ }^{1-i}$ While this type of analysis provides a succinct description of the expectations for a particular machine design, many of the critical physics parameters in the calculation must be evaluated by other means. These parameters describe characteristics such as profile shapes, hydrogenic density iraction, energy confinement time, etc. Ideally, one would use a transport code to estimate their values selfconsistently. The global calculation would then allow the entire operating space to be mapped out.

Howaver, at present reliable transport models for this task do not exist in fully validated form. So, we m'ist attempt to determine the input parameters for the glubal power balance using empirical knowledge. Because reactor designs are expected to operate at much higher plasma currents than recent experiments, it is uncertain how to extrapolate present results to the new regimes.

Clearly, uncertainty in the input parameters for the global power balance calculation translates into an uncertainty in the predicted reactor performance. Ho and Perkins ${ }^{8}$ recently proposed a method for quantitatively evaluating this uncertainty in reactor performance. Similar techniques have been described elsewhere. ${ }^{9,16}$ The result of the Ho and Perkins procedure is a "probability of ignition." The study reported here applies their methods to proposed designs of the Compact Ignition Tokamak ${ }^{11}$ (CIT). We will also examine brielly the Ignitor-U and ARIES designs as comparisons.

It must be emphasized that this approach to estimating the ignition probability has its foundation in the judgmental determination of the probabilities of achieving the individual input variables. Because of this somewhat subjective basis, the results should be used more for their value in providing an insight into the sensitivity to variations in plasma characteristics, such as profile peaking and plasma current, than for decisions related to the absolute value of the ignition probability.

We find (as did Ho and Perkins) that the probability of ignition is most sensitive to the parameters and scaling expressions that determine the energy confinement time. Consequently, we will focus attention on the level of 
uncertainty involved in extrapolating the present energy confinement time database to CIT. Reasonable ranges for the other critical parameters will be determined based upon experience with present experiments and expectations for CIT.

We have considered several parameter combinations for CIT: three different sets of magnetic field and plasma current specifications, all with about the same edge safety factor, and two contrasting operating regimes. The latter separates cases where flat density profiles are expected (for example. in the $\mathrm{H}$-mode ${ }^{12}$ ) from those in which peaked density profiles are obtained (for example, with pellets or strong inward particle pinch). This gives rise to six different configurations for CIT.

The results of our calculations indicate that CIT will have a substantial probability of ignition ( $\geq 40 \%$ ) if peaked density profiles can be obtained or if one of the two higher plasma current designs is used. In other cases, values of the energy multiplication factor $Q$ of order $\mathbf{1 0}$ are generally obtained. It is found that peaking the density profile yields about the same increase in the probability of ignition as raising the current by $2 \mathrm{MA}$. Finally, we have shown that the level of confinement in ignited cases is slightly less than that predicted by the Tang-Redi theory-based transport model ${ }^{13-13}$ evaluated at the same parameters. However, use of the Waltz, Dominguez, and Perkins model $^{6}$ in the global power balance calculation yields more pessimistic results.

In Sec. II, we will describe the global power balance calculation and the Monte Carlo procedure used to determine the probability of ignition. Section III provides a detailed discussion of the motivation for our choice of energy confinement time scaling; the ranges employed for the other parameters are specified in Sec. IV. We give our results in Sec. $V$ and compare with theory in Sec. VI. Finally, our conclusions will be presented in Sec. VII.

\section{Global Power Balance and Monte Carlo Calcula- tions}

A number of global power balance codes have been developed recently. ${ }^{2-7}$ They typically sclve an equation similar to

$$
P_{\alpha}+P_{O H}+P_{\text {aux }}=P_{\text {con }}+P_{\text {rad }} .
$$


The individual terms represent the volume-integrated contributions made to the total power balance by alpha, ohmic, and auxiliary heating; thermal conduction and radiated losses are on the right-hand side.

Codes such as the one to be described here are zero-dimensional (0-D) in that the plasma profiles are all specified on input. We will use for density, temperature, and plasma current density

$$
x=x_{0}\left(1-r^{2} / a^{2}\right)^{a_{2}},
$$

where $x$ is replaced by $\pi, T$, and $J$, respectively; $a$ is the plasma minor radius. Then, the alpha power is computed using

$$
P_{\alpha}=\eta_{\alpha} E_{0} 4 \pi^{2} R_{n} \int_{0}^{a} r d r n_{D} n_{T} \overline{\sigma \bar{v}} D T,
$$

where $\eta_{\alpha}$ is the alpha heating efficiency, $E_{\alpha}$ is the alpha particle energy, $R$ is the plasma major radius, and $\boldsymbol{x}$ is the plasma elongation. By taking $\eta_{s}<1$, we can include energetic al 5 ha particle losses. The reactivity, $\bar{\sigma} \bar{v}_{D T}$, is calculated with a formula obtained by Hively ${ }^{16}$ in order to ensure correct results in all temperature regimes. Consequently, this integral must be computed numerically for each value of the density-weighted, volume-averaged temperature, $\langle T\rangle \equiv\left\langle n_{e} T\right\rangle /\left\langle n_{e}\right\rangle$. The angle brackets here indicate a volume average.

The ohmic heating power is (all powers are in watts)

$$
\begin{aligned}
& P_{O H}=\frac{4.17 \times 10^{3} Z_{e f f} \ln A \gamma_{N C} T_{0}^{-3 / 2} V}{1+2 \alpha_{J}-\frac{3}{2} \alpha_{T}} \\
& \times\left\{\frac{B_{T}\left[1+\kappa^{2}\left(1+2 \delta^{2}-1.2 \delta^{3}\right)\right]}{2 \kappa R q_{0}}\right\}^{2},
\end{aligned}
$$

where $Z_{\text {eff }}$ is the effective charge, $\ln \Lambda$ is the Coulomb logarithm, $\gamma_{v c}$ is a neoclassical resistivity enhancement factor (constant, taken to be 2.5), $T_{0}$ is the central electron (and ion) temperature in $\mathrm{keV}, B_{T}$ is the toroidal magnetic field in tesla, $R$ and $a$ are in metres, $\delta$ is the triangularity, and $V$ is the plasma volume in $\mathrm{m}^{3}$. This expression for $P_{O H}$ is essentially the same as that used by Uckan. ${ }^{5}$ 
We will assume $\alpha_{J}=\frac{3}{2} \alpha_{T}$. and compute the safety factor on axis. $q_{0}=$ $q_{c y l} /\left(1+\alpha_{J}\right)$. For the equivalent cylindrical safety factor. we take

$$
q_{e y l}=\frac{5 a^{2} B_{T}}{R I_{p}} \frac{\left[1+\kappa^{2}\left(1+2 \delta^{2}-1.2 \delta^{3}\right)\right]}{2} .
$$

where $I_{p}$ is the plasma current in mega-amperes. These units will be used throughout this paper unless otherwise specified.

Only bremsstrahlung radiation is included ${ }^{5}$ in $P_{\text {rad: }}$ :

$$
P_{\text {rad }}=5.31 \times 10^{-37} \frac{n_{e 0}^{2} T_{0}^{1 / 2} Z_{e j,} V}{\left(1+2 \alpha_{n}+\frac{1}{2} \alpha_{T}\right)} .
$$

where $n_{e 0}$ is the central electron density.

Finally, the conducted losses are written as

$$
P_{\text {con }}=\frac{W_{\text {tot }}}{\tau_{E}}=\frac{2.40 \times 10^{-16}\left(\left(n_{e} T\right\rangle+\left\langle n_{i} T\right\rangle\right) V}{\tau_{E}} .
$$

We will use an $\mathrm{L}$-mode scaling expression for $\tau_{E}$ with a multiplier $C_{\boldsymbol{T}}$ indicating the level of enhancement above L-modes The particular scaling expression to be used and the value expected for $c_{T}$ will be discussed in Sec. III.

At a given $\left\langle n_{e}\right\rangle$ and $\langle T\rangle$, Eq. (1) is solved for $P_{\text {aur. }}$. The deuterium and tritium densities (assumed equal) are computed from $\left\{n_{e}\right\rangle$ using the input value of $Z_{\text {eff }}$ with carbon as the impurity species. If $T_{E}$ is a function of input power, this requires a numerical solution. On the other hand, if $\tau_{E}$ is expressed in terms of $\left\langle n_{e}\right\rangle$ and $(T\rangle$, Eq. (I) can be solved explicitly.

The density and $\beta$ limits restrict the range of $\left\langle n_{e}\right\rangle$ and $\langle T\rangle$. In particular, we assume a density limit of the Hugill form, ${ }^{17}$

$$
\bar{n}_{e, \max }=h \frac{B_{T}}{R q_{c y l}} 10^{20} \mathrm{~m}^{-3}
$$

the over-bar denotes a line average. Other scalings have been found in more recent experiments ${ }^{18,19}$; however. at fixed $\kappa$ and $\delta$, they can be rewrittes in the form of Eq. (8). The value of the multiplier $h$ will be determined using these various experimental results (see Sec. IV.F). A Troyon scaling is assumed for the $\beta$ limit ${ }^{20.21}$ :

$$
\beta_{\max }=g_{p} \frac{I_{p}}{a B_{T}} \% .
$$


We will discuss our choice for the multiplier $g_{B}$ in Sec. IV.E.

There are a total of seven as-yet-unspecified parameters in the O-D calculation: $\alpha_{n}, \alpha_{T}, \eta_{\alpha}, Z_{e_{j}^{\prime} \rho}, c_{r}, h$, and $g_{\beta}$. The value of each in the reactor regime is uncertain. It is this uncertainty that motivates the development of a Monte Carlo approach ${ }^{8}$ to solving $\mathrm{Eq}$. (1), yielding a probabilistic description of the performance of the reactor.

As was done by Ho and Perkins, ${ }^{8}$ we will use the ignition margin to characterize reactor performance:

$$
H_{I} \equiv \frac{P_{a}}{P_{\text {con }}+P_{\text {rad }}}
$$

This is related to the more familiar $Q \equiv 5 P_{a} /\left(P_{O H}+P_{a u r}\right)$ by

$$
Q=\frac{5 M_{I}}{1-M_{I}}
$$

The ignition margin is preferred since it is well-behaved in the ignited regime $\left(M_{I} \geq 1\right)$.

The Monte Carlo calculation is performed as follows ${ }^{8}$ :

1. Assign to each of the seven critical parameters a probability distribution spanning the range of values anticipated in the actual experiment.

2. Sample one complete set of parameters from the given probability distributions using a random number generator.

3. Compute the maximum value of $M_{l}$ for this set of parameters subject to the density and $\beta$ limits.

4. Repeat steps 2 and 3 until the statistical fluctuations are insignificant.

For all of the cases to be discussed here, 5000 iterations are used. In order to plot the resulting $M_{I}$ distribution, it is divided into bins of width $\Delta M_{I}=$ 0.05 . By dividing the number of cases in each bin by 5000, we obtain a normalized probability distribution for $M_{l}$. In addition, we will plot the integral of the distribution above a given $M_{I}$. The value of the latter at $M_{I}=$ 1 is then defined as the probability of ignition. ${ }^{8}$ Similarly, the probability of 
doing better than $Q=10$ is just the value of the integral of the probability distribution above $M_{t}=0.67$.

For scaling expressions of the form $\tau_{E} \propto\left\langle n_{e}\right\rangle^{\xi} P_{\text {input }}^{-r}$, where $P_{\text {input }}=$ $P_{\alpha}+P_{O H}+P_{a u x}-P_{\text {rad }}$ is the net input power, it can be shown that

$$
\frac{\partial M_{I}}{\partial\left\langle n_{\varepsilon}\right\rangle}=\frac{M_{I}}{\left\langle n_{e}\right\rangle} \frac{P_{\text {con }}}{P_{\text {con }}+P_{\text {rad }}}\left(\frac{1-2 \gamma+\xi}{1-\gamma}\right)
$$

This implies that when : $-2 \gamma+\xi>0$ and $\gamma<1$ (true for many L-mode scaling expressions), $M_{I}$ is monotonically increasing with density. So. to determine the maximum value for a given set of the critical parameters, we need only search over $\langle T\rangle$, using the maximum density allowed by Eqs. (8) and (9).

\section{Energy Confinement Time Formulation}

It would be most desirable to use theoretical models calibrated against experiment to carry out the extrapolation of present results to future devices. We will compare our results for CIT with those predicted by specific models $^{6,13-15}$ in Sec. VI. While models of this sort have had some success in matching experiment, no single theory has been found reliable enough to be used as the sole basis for predicting the performance of future experiments.

There have been only a handful of dimensionless parameters used by theorists to describe transitions from one type of physical behavior to another in tokamaks (see Table I). The value of each that is required in order for CIT to ignite is in a range that has been experimentally explored. We conclude that there are no apparent theoretical boundaries to be crossed in extrapolating from present data to CIT. This increases our confidence in being able to predict the value of $\tau_{E}$ for L-mode operation of CIT from the present L-mode database.

The L-mode database ${ }^{22}$ consists of energy confinement time data that have been steadily collected over the last several years from many tokamaks. The values of $\tau_{E}$ span two orders of magnitude $\left(\sim 10^{-2}-1 \mathrm{~s}\right)$; the minor radii of the devices included range from $\sim 0.3 \mathrm{~m}$ to $>1 \mathrm{~m}$. Ohmic and so-called enhanced confinement (e.g., H-mode) discharges are excluded. The result is a set of 1300 data points describing the minimum level of energy confinement 
that can be expected in an auxiliary heated tokamak (no distinction is made between neutral beam and radio-frequency heating).

It is well known that CIT (and other reactor designs as well: see. for example, Ref. 5) will not ignite with strictly L-mode confinement. The level of improvement over $\mathrm{L}$-mode required is within that attainable in present devices operated in enhanced confinement regimes. Well-established examples are the $\mathrm{H}$-mode, ${ }^{12}$ supershot neutral beam heating, ${ }^{23}$ and pellet injection. ${ }^{2+}$ It would be most desirable to be able to predict CIT performance for each of these regimes. However, detailed theoretical explanations do not exist for any oi them. and their individual databases are too sparse to allow reasonable certainty in extrapolating to CIT.

All we can do with the present data is to use them to prescribe just how much enhancement above L-mode can be expected. In other words. we will show below that we can extrapolate the value of $\tau_{E}$ in L-mode. $\tau_{E . L}$, to CIT with reasonably small statisiical uncertainty. Then, we argue that since virtually every recent tokamak has developed some means of enhancing confinement above L-mode, CIT should be able to do so as well. So, we express the energy confinement time for a generic enhanced regime as

$$
\tau_{E}=c_{\tau} \tau_{E, L}
$$

and allow recent experience with these various modes of operation to dictate a range of values for $c_{\tau}$. There is strong evidence indicating that at least the $\mathrm{H}$-mode energy confinement time can be written in this way. ${ }^{22}$

\section{III.A. Regression Fits to L-mode Database}

We now use the standard approach of fitting the L-mode energy confinement time with a power law expression. ${ }^{22,25}$ Namely, the scaling is assumed to be of the form

$$
\tau_{E, L}=e^{\delta_{1}} \bar{A}_{i}^{\delta_{3}} \kappa^{b_{3}} I_{p}^{b_{4}} \bar{n}_{e}^{b_{s}} B_{T}^{b_{s}} a^{\delta_{7}} R^{\delta_{3}} P_{\text {inpul }}^{b_{g}}
$$

where $\bar{A}_{i}$ is the effective ion mass in AMU, $\bar{n}_{e}$ is the line-averaged electron density, and the nine coefficients $b$, are determined by performing a simple linear regression fit to In $\tau_{E, L}$. We will label the set of best fit coefficients as 
$\dot{b}_{j}$. For more details on procedures that have been used previously and on other ways of writing the parametric dependence of $\tau_{E}$, see Refs. 22 and 25 .

In order to determine whether devices of different sizes will yield different predictions of $\tau_{E, L}$ for CIT, the database is divided into three parts labeled: "small" $(a<0.35 \mathrm{~m})$, "medium" $(0.35<a<0.6 \mathrm{~m})$, and "big" $(a>0.6$ m). As an example, we show in Fig. 1 a plot of the regression formulat Eq. (14) fit to the "medium" and "big" segments $\left(\tau_{E . L}^{M B}\right)$ against all of the data $\left(\tau_{E}^{E X P}\right)$. The quality of the fit is good over the entire range of $\tau_{E . L}$. For the rest of our discussion, we will focus on only the combined "medium" and "big" database. Since this is a $\log$-log plot, it is difficult to quantify the scatter of the data about the fit. Conseque , uly, we present in Fig. 2 a frequency distribution of $\ln \left(\tau_{E}^{E X P} / \tau_{E . L}^{M g}\right)$. We can see here that the errors are distributed in an approximately normal fashion. The width of the best-fit gaussian shown in the figure is essentially the same as that of the histogram. corresponding to about $11 \%$ error at the $1 \sigma$ level.

\section{III.B. Extrapolating I-mode Confinement to CIT}

Standard texts in linear regression analysis give the uncertainty, $\sigma_{e x}$, in predicting a future observation from a fit as ${ }^{26}$

$$
\sigma_{e x}=\tilde{\sigma}\left[1+\boldsymbol{x}_{0}^{\prime}\left(\boldsymbol{X}^{\prime} \boldsymbol{X}\right)^{-1} \boldsymbol{x}_{0}\right]^{1 / 2},
$$

where $x_{0}$ is the column vector of the regressor variables (i.e., $I_{p}, R, \bar{n}_{t}$. etc.) for the future observation. $\boldsymbol{X}$ is the $n \times \boldsymbol{p}$ matrix of the data regressor variables ( $n$ sets of observations of the $p-1$ regressor variables; here $n \simeq 1800$ and $p=9$ ), and $\hat{\sigma}^{2}$ is the residual mean square error from the regression fit. The prime (') indicates the transpose. Clearly, there are two contributions to $\sigma_{\mathrm{ex}}$. The first (represented by the " $1 "$ ) is just the variation of the data about the fit. Namely, if the coefficients obtained by the fit did indeed represent the actual parametric dependence of $\tau_{E . L}$, then the uncertainty for the extrapolated point would equal the standard deviation of the data about the fit. We will take $\dot{\sigma}=11 \%$ as our estimate for this (see Fig. 2).

The second contribution arises from the error in the fitted model. In other words, this term comes from the fact that we do not know with certainty that L-mode scaling in CIT will look like Eq. (14) with our fit values $\hat{b}_{j}$. Note 
that this factor is minimized when $x_{0}$ falls within the range of the existing data. It is instructive to estimate its contribution as follows.

We know (see Ref. 26 for example) that the $100(1-\mu) \%$ joint confidence region for the $p$ regression coefficients $b_{j}$ is a $p$-dimensional hyper-ellipsoid described by the inequality

$$
\frac{(\mathbf{b}-\hat{\mathrm{b}})^{\prime} \boldsymbol{X}^{\prime} \boldsymbol{X}(\mathbf{b}-\hat{\mathbf{b}})}{p \hat{\sigma}^{2}} \leq F_{\mu, p, n-p}
$$

where $F_{4,0, n-p}$ is the standard $F$-distribution. ${ }^{26}$ Equation (16) gives the uncertainty in the fit coefficients [at the $100(1-\mu) \%$ confidence level] due to the scatter in the data. We can get an idea of what this joint confidence region is by randomly generating sets of $b_{i}$ (say, within two standard deviations of $\left.\dot{b}_{j}\right)$, testing them using Eq. (16), and plotting the ones that satisfy it.

Doing this with $\mu=0.33$, a $1 \sigma$ confidence level, for the combined "medium" and "big" portions of the L-mode database, we generate 1000 sets of coefficients. In Fig. 3, we show the projection of the resulting hyperellipsoid onto the $b_{7}-b_{8}$ (exponents on $a$ and $R$ ) plane. It is clear from Fig. 3 that we know $b_{7}+b_{8}$ (length scaling of $r_{E}$ ) with much greater certainty than we know $b_{7}-b_{8}$ (aspect ratio scaling). This is due to the inclusion of a large range of device sizes in the L-mode database, but only small variations in aspect ratio, $R / a=2.5-4$.

Figure 4 demonstrates a modest correlation between the $I_{p}$ and size scaling exponents. This arises because the plasma current capability of iokamaks has generally increased with size. Contrary to this trend, CIT is designed for high current and only moderate size. This would seem to indicate some uncertainty in predicting $\tau_{E, L}$ for CIT. As is clear from Fig. 4, however, the range of $I_{p}$ exponents within this $67 \%$ joint confidence region is relatively small. So, the actual error due to this effect is not overwhelmingly large. All of these considerations are properly accounted for in Eq. (15).

Now, we can take these 1000 scaling expressions and calculate what each predicts for $\tau_{E_{\mathrm{r}} L}$ in CIT. Assuming "standard" CIT parameters (see Sec. V) $R=2.1 \mathrm{~m}, a=0.65 \mathrm{~m}, \kappa=2, B_{T}=10 \mathrm{~T}, I_{p}=11 \mathrm{MA}, \bar{n}_{\varepsilon}=5 \times 10^{20} \mathrm{~m}^{-3}$, $\bar{A}_{i}=2.5$, and $P_{\text {input }}=100 \mathrm{MW}$, we obtain Fig. 5. The result is $\tau_{E . L}^{M B}=0.32$ $\mathrm{s} \pm 5 \%$.

This extrapolation prror of $5 \%$.is essentially what we obtain by evaluating the second term of Eq. (15) directly. This is much smaller than the $11 \%$ 
error due to the scatter in the data about the fit, so that when summed in quadrature, the total error is still $\sigma_{\text {ex }} \simeq 11 \%$.

We should make some comments about this result. One reason $\sigma_{e x}$ is small is that the range of extrapolation to CIT is not great, particularly in a logarithmic sense. Of the most important scaling variables, only input power exceeds the capabilities of present devices by more than a factor of two. The density and magnetic field are also well above the values represented in the database. Since the scaling of $\tau_{E}$ with these variables is weak, however, their impact on the extrapolation is not great. On the other hand, there are no similar high density, high field data in the database, as is demonstrated by Fig. 4 (although this did not degrade the above extrapolation error estimates significantly). Such a lack of variation in certain directions in parameter space. evidenced also by Fig. 3, may lead to a near degeneracy of the data matrix ${ }^{27}$ ("colinearity"). In this case, the coefficients $b$ would be very sensitive to small errors in the data. Advanced regression techniques such as principal component analysis and ridge regression can be used to produce more stable results. ${ }^{2 \pi}$ The most desirable cure is to add data in the problem areas. ${ }^{27}$

Another reason $\sigma_{e x}$ is only $\sim 11 \%$ is that $n \simeq 1300$ is large. That is, $\sigma_{e x}$ scales like $n^{-0.5}$. It has been suggested, however, that simply lumping all of the tokarnak data together may not be the proper approach. ${ }^{27}$ This would be the case, for instance, if sources of systematic error in the data varied from tokamak to tokamak. Again, more advanced techniques would be required to handle this situation correctly. ${ }^{2 T}$ Such detailed analyses are beyond the scope of the present work.

We used only the "medium" and "big" segments of the database above in order to look at devices closest in size to the planned CIT. However, using the entire database ("sntall." "medium," and "big") does not produce a significantly different answrr. We now compare these values with those predicted by other recently obtaimed scaling expressions at these same standard CIT parameters. We consirler Goldston ${ }^{28}(\mathrm{G})$, Kaye-Goldston ${ }^{25}(\mathrm{KG})$, and Kaye-All-Complex ${ }^{22}$ (KAC):

$$
\begin{aligned}
\tau_{E, L}^{G} & =0.0302 \vec{A}_{i}^{0.5} i^{0.5} I_{p} a^{-0.37} R^{1.75} P_{\text {input }}^{-0.5} \\
& =0.32 \mathrm{~s}
\end{aligned}
$$




$$
\begin{aligned}
{ }_{E . L}^{K G G} & =0.025+\vec{A}_{i}^{0.5} \kappa^{0.28} I_{p}^{1.24} \vec{n}_{e .19}^{0.26} B_{T}^{-0.09} a^{-0.49} R^{1.65} P_{\text {input }}^{-0.58}, \\
& =0.62 \mathrm{~s}, \\
T_{E . L}^{K . A C} & =0.0521 \vec{A}_{i}^{0.5} \kappa^{0.25} I_{p}^{0.85} \vec{n}_{e .19}^{0.1} B_{T}^{0.3} a^{0.3} R^{0.85} P_{\text {input }}^{-0.5}, \\
& =0.37 \mathrm{~s}:
\end{aligned}
$$

$P_{\text {input }}$ is in megawatts, and $\bar{n}_{e, 19}$ is the line-averaged electron densit:" in units of $10^{19} \mathrm{~m}^{-3}$. The Kaye-All-Complex matches the result obtained from the medium-big database closely, both in magnitude for these particular parameters and in scaling. This is not surprising since $\tau_{E . L}^{K A C}$ is derived from the same database. although using somewhat different techniques. The other scalings were obtained using older and less extensive databases. Consequently, we vill take $\tau_{E, L}^{K} A C$ as given in Eq. (1i) to be our basic L-mode confinersent time.

\section{III.C. L-mode Enhancement Factor}

For the parameters used above to arrive at $\tau_{E . L}^{K . A C}=0.37 \mathrm{~s}$, a steady state would require $P_{\text {con }}=100 \mathrm{MW}$. Thus, by Eq. ( 7$)$. $W_{\text {tot }}=37 \mathrm{MJ}$. This corresponds to $\langle T\rangle \simeq 5 \mathrm{keV}$ if $\left\langle n_{e}\right\rangle \simeq 5 \times 10^{20} \mathrm{~m}^{-3}$. A quick estimate of of the alpha power at this density and temperature (see Ref. 5 for example) yields $P_{\alpha} \simeq 30 \mathrm{MW}$. Clearly then, this is not an ignited scenario for CIT. It has been pointed out elsewhere ${ }^{5}$ that CIT will not ignite in general with purely L-mode confinement. We will later describe circumstances in which it does.

Thus, we must examine means of enhancing confinement above L-mode. $\mathrm{H}$-mode is considered to be the most likely means of doing this in CIT primarily because $\mathrm{H}$-mode operation has been achieved on 11 tokamaks to date. including all divertor tokamaks ${ }^{29,30}$ : JET, ASDEX, DIIT, DIII-D, PDX, PBX, PBX/M, TFTR, JFT-2M, JT-60, and JIPT-II. Furthermore, H-mode has been obtained with neutral beam. radio-frequency, and even ohmic heating. ${ }^{29}$ There appears to be a minimum density $\left(\sim 2 \times 10^{19} \mathrm{~m}^{-3}\right)$ as well as a minimum total heating power required for entry into the $\mathrm{H}$-mode regime. ${ }^{29}$ Evidence was presented at the meeting referred to in Ref. 29 that this threshold power scales like $B_{T} \times$ plasma surface area. When evaluated for CIT, this scaling suggests ${ }^{30}$ that the required $P_{\text {aur }} \sim 10-30 \mathrm{MW}$.

However, $\mathrm{H}$-mode data are still sparse enough to allow only an estimate 
of the multiplier in

$$
\tau_{E}=c_{\top} \tau_{E, L}^{K A C} \text {. }
$$

Kaye has carried out a preliminary analysis of the $\mathrm{H}$-mode database ${ }^{22}$ and has found that parametric trends of L-mode scaling are followed at least roughly. Furthermore, he arrived at an estimate of $c_{r} \simeq 2.5$. Values as large as 3 have been claimed for more recent experiments. ${ }^{39}$ This level of enhancement is similar to that routinely attained in TFTR supershots. ${ }^{23}$ So, in defining the probability distribution of $c_{\tau}$ for input to the Monte Carlo code, we will take $c_{\tau}=\mathbf{2 . 5}$ as an upper limit.

We choose a lower limit of $c_{r}=1$. The factors contributing to this range of $c_{r}$ are (i) the uncertainty in estimating $c_{r}$ for present enhanced confinernent experiments, (ii) the uncertainty in scaling these values to CIT, and (iii) the uncertainty in scaling $\tau_{E . L}^{K .} \cdot C$ as discussed in Sec. IIL.B. For simplicity, we take the resulting probability distribution to be flat between $c_{,}=1$ and 2.5 .

We would like to make two additional points. First, $\tau_{E, L}$ is not combined in an inverse square sense with a neo-Alcator scaling $\tau_{N A}$ as has been the practice in the past. ${ }^{5.28}$ The reason for this is that the L-mode database contains many points at low power; $\tau_{E, L}^{K} H_{C}^{C}$ should be accurate in those regimes. ${ }^{31}$ Furthermore, since the operating points we will be considering are typically at the intersection of the density and $\beta$ limits, the irput power is substantial so that $\tau_{E, L}^{K} \mathcal{L}^{C} \ll \tau_{N A}$. In this case, it does not matter whether or not the inverse square combination is used.

Secondly, $T_{E, L}^{K A C}$ as originally derived is a function of input power. It is more reasonable from a physical point of view to have $r_{E}$ scaling with only thie bulk plasma parameters, ${ }^{28}$ i.e., $\left\langle\pi_{e}\right\rangle$ and $\langle T\rangle$. We can accomplish this by inserting our expression for $\tau_{E}$ into Eq. (1) to obtain $P_{\text {input }}=P_{\alpha}+P_{O H}+$ $P_{a u x}-P_{\text {rad }}$ as a function of $W_{t o t}$ and then substituting it into $r_{E}$. With $\tau_{E}=f_{\tau} P_{\text {inpus }}^{-7}\left(f_{\tau}\right.$ contains all of the non-power dependence including the multiplier $\left.c_{\tau}\right)$, this yields $\tau_{E}=\left(f_{r} W_{\text {tot }}^{-\gamma}\right)^{1 /(1-\gamma)}$. In effect, we have solved Eq. (1) in advance so that we can compute $P_{\text {aur }}$ explicitly from Eq. (1). 


\section{Critical Parameter Probability Distributions}

We now discuss the probability distributions for the six other critical parameters in the 0 - $D$ calculation. These are intended to represent the values obtained simultaneously in CIT"s "best" shots. We mean this in the sense that these parameters would be achieved in hundreds of tokamak shots. but not necessarily the thousands of full power pulses available to CIT, nor in only a very few anomalously good discharges.

\section{IV.A. Density Profile Exponent, $\alpha_{n}$}

We will be considering two separate $\alpha_{n}$ distributions. The first, referred to hereafter as the "flat" case, presumes gas fueling or operation in the H-mode regime. It is well known that density profiles in the H-mode can be completely flat and even inverted. Since inverted profiles are not easily handled by the present formalism, we disregard that case and take $0<\alpha_{n}^{\text {flat }}<0.75$ as a reasonable rarige. As will be the case with ali of the other parameters (except $g_{\beta}$, see below), we use a uniform probability distribution; there are no clear physical reasons for doing otherwise.

The other $\alpha_{n}$ distribution will be referred to as "peaked." In this case, we assume that a peaked density distribution can be formed and heated effectively. This may be accomplished by early pellet injection if the profile can be maintained as the plasma heating proceeds. If central fueling is required throughout the discharge, provisions will have to be made for deeply penetrating pellet injection, possibly aided by a density self-pinching effect. Recent experience with pellets and supershots ${ }^{23}$ indicates that peaking factors of $n_{0} /(n)=2.5-4$ are possible, corresponding to $1.5<\alpha_{n}^{\text {peaked }}<3$, the range assumed in the present study. In this case, it might be argued that nonparabolic profiles should be used, but such refinements are outside the scope of a simple 0-D calculation and are better addressed by more detailed 1. or 1-1/2-D transport codes.

\section{IV.B. Temperature Profile Exponent, $\alpha_{T}$}

We decide upon the expected range of values of $\alpha_{T}$ in the following way. First, in resistive steady-state with classical resistivity we have $J \propto T^{3 / 2}$. So, 
$J_{0} /\langle J\rangle=1+\alpha_{J}=1+\frac{3}{2} \alpha_{T}$. This then implies $q_{c y l} / q_{0}=1+\frac{3}{2} \alpha_{T}$. Taking $q_{0}=1$ (e.g., with sawtooth oscillations), and $q_{\text {cyl }} \simeq 3$, we have $\alpha_{T} \simeq 4 / 3$.

The temperature profile could be more peaked than this, for example, with sawtooth stabilization or with highly peaked heating profiles. It could also be flatter due to the elongation, $k=2$, planned for CIT. Choosing a mean value of $\alpha_{T}=1.25$ via the argument of the previous paragraph, and taking the other considerations into account in determining the range of $\alpha_{T}$. we arrive at a flat probability distribution $0.75<\alpha_{T}<1.75$.

\section{IV.C. Alpha Heating Efficiency, $\eta_{a}$}

We will assume that the magnetic field ripple in CIT has been made low enough that there is very little ergodic banana loss due to external ripple. However, there may be some lost due to MHD activity at the highest 3 . So. we take the alpha heating efficiency to be close to unity, $\eta_{\alpha}=0.95$, without assigning any width to the distribution.

\section{IV.D. Effective Charge, $Z_{\text {eff }}$}

Judging from previous high field, low power machines, we might expect $Z_{\text {ejf }}$ to be as low as 1.3. But, in higher power, lower field machines (with carbon walls), one could be limited even in the best shots to $Z_{e f f}=2$. So, we take $1.3<Z_{\text {eff }}<2$.

For simplicity, we will assume that all of the impurities are carbon. If we concern ourselves only with the chances of achieving ignition, we can neglect helium ash build-up. We can consider the alterations made by residual helium ash or metallic impurities in the discharge as being accounted for by this range of $Z_{\mathrm{e}\} \text {. }}$.

\section{IV.E. Troyon Coefficient, $g_{\beta}$}

DIII-D has operated stably ${ }^{32}$ with $g_{\beta}$ as high as 3.5. Other machines seem to be limited to values as low as $g_{B} \simeq 2.5$. There may be additional problems associated with alpha particles but, on the other hand, the understanding and control of these instabilities may improve between now and when CIT 
is actually built. This leads us to consider an overall range of $2<g_{g}<3.5$. but we consider $2.5<g_{3}<3$ to be the most likely region of operation. For this reason. we employ a trapezoidal distribution as shown in Fig. 6 (a).

The density and, hence, pressure profiles assumed in the "peaked" case zre quite different from those arising in optimization studies. ${ }^{20.21}$ Hence, we find it prudent to use the distribution shown in Fig. 6 (a) only for the flat profile case and to reduce $g_{s}$ by 0.5 overall for peaked density profiles [see Fig. $6(b)]$.

\section{IV.F. Hugill Coefficient, $h$}

Our form for the density limit, Eq. (8), is motivated by the early work on the subject ${ }^{17}$ and assumptions made in previous discussions of $\mathrm{CIT} .{ }^{33}$ At that time,$^{33} h=1.5$ was considered an effective description of the data. The actual experimental observation is that the maximum density increases with current. But, $q_{c y l}$ also depends strongly on plasma elongation. Only recently has sufficient data become available to test whether or not this elongation dependence is correct.

Greenwald ${ }^{19}$ has examined a large amount of data from several different tokamaks and has concluded that the expression

$$
\bar{n}_{e, \text { max }}^{\text {Greerwald }}=\frac{I_{p}}{\pi a^{2}} 10^{20} \mathrm{~m}^{-3}
$$

does the best job of bringing the data into line; Marfes appear at about 60 - $80 \%$ of this value. More recent experiments on DIII-D have verified this result $^{34}$ for elongations up to $\kappa=2$.

On the other hand, it has been found ${ }^{18}$ in JET beam-heated discharges that

$$
\bar{\pi}_{\mathrm{max}}^{\mathrm{N}}=\frac{2 B_{T}}{R q_{e}} 10^{20} \mathrm{~m}^{-3},
$$

where $q_{e}=5 a^{2} \kappa B_{T} / R I_{p}$ is the engineering $q$-value. This limit also works reasonably well for ohmically heated cases with pellet injection. ${ }^{18}$

All three of these have the same basic structure $\bar{n}_{e, \max } \propto I_{p} / a^{2}$. So. at fixed $\kappa$ we can express the Greenwald and JET formulas as in Eq. (8) by using appropriate values of $h$. We find that for CIT $(\kappa=2$ and $\delta=0.4)$, 
$h^{J E T}=3$ and $h^{\text {Greenwald }}=4.75$. If we reduce the latter to about $80 \%$ of Eq. (19) in order to prevent Marfes, we get $h^{\text {Greenwald }} \simeq 4$.

Considering the range of these results and the uncertainty in scaling to CIT, we propose a probability distribution (uniform) with $1.5<h<4$. We have not taken into account here the possibility that the density limit increases with power. ${ }^{35}$

Finally, we sumrarize our proposed ranges for the seven critical parameters in Table II. There exist argumenis for having cross-correlations between some of the probability distritutions (e.g., $Z_{\text {eff }}$ decreasing with increasing $h$ ). However, we find that the value of $c_{7}$ is so crucial and its uncertainty so great that the details of the other parameter distributions have only a weak effect on the probability of ignition. On the other hand, the mean values of all of the parameters are important.

\section{Results}

We have examined three different magnetic configurations for CIT:

1. $I_{p}=9 \mathrm{MA}, B_{T}=8.2 \mathrm{~T}-$ present CIT design with existing TFTR power supplies.

2. $I_{p}=11 \mathrm{MA}, B_{T}=10 \mathrm{~T}$ - present CIT design with upgraded power supplies.

3. $I_{p}=13 \mathrm{MA}, B_{T}=11.8 \mathrm{~T}-$ possible enhanced design using a bucked coil design.

For all three designs, $R=2.1 \mathrm{~m}, a=0.65 \mathrm{~m}, \kappa=2$, and $\delta=0.4$. The magnetic fields were chosen in cases (1) and (3) to yield $q_{c y l}=2.73$ as in case (2). Combined with the two density profile options, we have a total of six CIT configurations to consider. With this choice of parameters, we can compare the relative merits of increasing the current by $2 \mathrm{MA}$ and changing the density profile shape from flat to peaked.

In Fig. 7 we show the results of our calculation for the three cases with flat density profiles. Both the $M_{I}$ probability distribution (a) and the integral of the distribution above a given $H_{I}(\mathrm{~b})$ are shown. The correspording 
results for peaked profiles are shown in Fig. 8 . We summarize these plots in Tables III and IV where we give the probability of ignition. $P\left(M_{I}>1\right)$, and probability of doing better than $Q=10, P\left(M_{T}>0.6 T\right)$, respectively.

According to these calculations, CIT has a $\geq 40 \%$ probability of ignition with that density profiles if either of the two upgraded magnetic configurations can be obtained or in the baseline magnetic configuration if peaked density profiles can be achieved. The likelihood of being able to perform significant alpha physics research $(Q>10)$ is better than about $40 \%$ for all six machines and is a near certainty with the magnetic upgrade or the peaked density profiles. If the $L$-mode enhancement factor is held fixed at $c_{T}=1$ in the 1.3 MA peaked profile case. $P\left(W_{I}>1\right)$ drops to $\simeq 1 \%$. That is, in this case. there even exists a slim chance of ignition with purely L-mode confinernent. But. $P(Q>10)=56 \%$ in this situation.

It is apparent from both Tables III and IV that peaking the density profile (as described in Sec. IV.A) is about equivalent to raising the plasma current by $2 \mathrm{MA}$. One consideration not entering this discussion explicitly is the expected improvement in energy confinement with peaked density profiles; ${ }^{15}$ it is implicitly included in the choice of $c_{\tau}>1$.

$U_{p}$ to this point, we have not concerned ourselves with the value of $P_{a x x}$ required in these various calculations. It is critical since only $10-25 \mathrm{MWW}$ are planned at present. Fortunately, the power needed in ignited cases to cross the saddle point in $\left\langle n_{e}\right\rangle$ and $\langle T\rangle$ space (see Ref. 5 and references therein) from ohmic equilibrium to the ignition window is generally $<10 \mathrm{MW}$. To allow for the thermal inertia of the plasma and the finite pulse length of CIT (5 s flat-top), $P_{\text {aux }}=20 \mathrm{MW}$ is desirable. ${ }^{35}$ For slightly subignited cases. $P_{\text {aux }}=20 \mathrm{MW}$ would allow driven operation at high $Q$ (e.g., $Q>10$ ).

In Fig. 9, we show a pessimistic scenario. These are the contours of constant $P_{\text {aus }}$ obtained from Eq. (1) in the $\left(n_{e}\right\rangle-\langle T\rangle$ plane for the $9 \mathrm{MA}$ case with flat density profiles; all parameters have been evaluated at the means of their respective distributions. While $P_{\text {sux }} \sim 50 \mathrm{MW}$ is required to reach the maximum $Q$ allowed by the density and $\beta$ limits $(Q \sim \tau)$, we can approach $Q \simeq 6$ with just the $25 \mathrm{MW}$ of auxiliary heating planned for CIT. Thus, even in this case, the prospects for doing significant alpha physics research look fairly good with the planned auxiliary power capability. 
In a situation where $Q=10$ is the best performance that can be attained at arbitrary $P_{\mathrm{aux}}$, a similar argument applies. as is demonstrated in Fig. 10. This plot is the sarne as Fig. 9. but for the 11 MA design. Here, however. $c_{\text {. }}$ is reduced to 1.46 to obtain a case in which $Q=10$ is just at the density limit. We see that in this instance, $25 \mathrm{MW}$ of auxiliary heating would allow us to reach $Q \simeq 8$.

We now apply this same procedure to two other proposed reactor designs. With a few exceptions, we employ the same input probability distributions as for CIT so that the machines can be compared on an equa! basis. However. it may be inappropriate to evaluate each of the designs in this way since the mode of operation planned for them may differ considerably from that of CIT.

We consider one ARIES design ${ }^{37}: R=6 \mathrm{~m}, a=\mathrm{Im} . \kappa=2.2, \delta=0.5$. $B_{T}=14.7 \mathrm{~T}$, and $I_{p}=8.2 \mathrm{MA}$. In this case, we take $q_{0}=1.8$ and increase the mean value of $\alpha_{T}$ to $1.5,1<\alpha_{T}<2$. All of our other assumptions are in line with those being used in ARIES modeling elsewhere. ${ }^{37}$ We find $P\left(M_{I}>1\right)=48 \%, P(Q>10)=70 \%$ for the peaked profile case, and $P\left(M_{I}>1\right)=15 \%, P(Q>10)=46 \%$ for the flat case. Note that neutral beam injection is planned for ARIES so that a means of peaking the density profile not present in the proposed CIT is available.

The Ignitor- $U$ experiment ${ }^{38}$ has two possible scenarios for its operation. In the first, the field and current are ramped up to $13 \mathrm{~T}, 12 \mathrm{MA}$ transiently before being brought down to flattop values of $10 \mathrm{~T}, 9.4 \mathrm{MA}$. The second scenario involves ramping up directly to flattop parameters of $11 \mathrm{~T}, 10$ MA. We will evaluate the probability of ignition for the two parts of the first scenario only; the second scenario should yield results intermediate between them. In all cases, $R=1.12 \mathrm{~m} . a=0.43 \mathrm{~m} \quad \kappa=1.8$, and $\delta=0.33$.

During the transient high-field state, $P\left(M_{I}>1\right)=96 \%$ and $76 \%$ for peaked and flat density profiles. respectively. The ignition probability is still quite significant, $67 \%$ (peaked) and $42 \%$ (flat) for the $10 \mathrm{~T}, 9.4$ MA Hattop.

Such high levels of performance are needed in Ignitor as it is designed to achieve ignition with little or no auxiliary heating. ${ }^{38}$ One reason Ignitor fares so much better than CIT is that the former has a higher density limit $\left(\propto I_{p} / a^{2}\right)$ and a higher $\beta$ limit $\left(x I_{p} / a B_{T}\right)$. However, as a result $q_{c y l}$ is significantly lower, $q_{c y l}=2.2$. The observation of degraded confinement in 
$\mathrm{H}$-mode discharges in DIII- $\mathrm{D}^{39}$ for edge $q$ values below 3 and the increased risk of disruption at low edge $q$ have motivated the requiremant of a higher $q_{c y l}$ value for CIT.

\section{Comparison with Theory}

We now determine how the above results, obtained by extrapolating empirical data and experience, compare with the predictions of two specific theoretical models. For the first one, we will focus on the level of confinement required at ignition. Rather than using a global parameter such as $\tau_{E}$ for the comparison, we will look at the local thermal diffusivities.

For a cylindrical equilibrium with elongated (constant $\kappa$ ) cross section, the diffusion equation equivalent to $\mathrm{Eq}$. (1) is ${ }^{2}$

$$
Q_{\mathrm{rad}}-Q_{\alpha}-Q_{O H}-Q_{\mathrm{auz}}=\frac{1}{r} \frac{\partial}{\partial r}\left[\frac{\left(1+\kappa_{*}^{2}\right)}{2 \kappa^{2}} r\left(n_{e}+n_{i}\right) \times \frac{\partial T}{\partial r}\right],
$$

where $\chi$ is the thermal diffusivity, $\kappa_{.}^{2}=\kappa^{2}\left(1+2 \delta^{2}-1.2 \delta^{3}\right)$, and

$$
4 \pi^{2} R \kappa \int_{0}^{a} r d r Q_{x}=P_{x}
$$

relates the local terms of Eq. (21) to the global terms of Eq. (1). Since the density and temperature profiles are specified, we can immediately find $Q_{\text {rad }}$, $Q_{\sigma}$, and $Q_{O H}$. We assume that $Q_{a u z}$ has a profile shape identical to that of $n_{e}(r) T(r)$. This apparently arbitrary choice is motivated by the fact that $P_{\text {aus }}$ can be viewed not only as the power required to maintain steady state, but also as the time-rate of change of the plasma energy in the aosence of auxiliary power, $\partial W_{\text {sot }} / \partial t$.

We integrate Eq. (21) once and solve for $x(r)$. Typical values of $x(r=$ $a / 2)$ and their standard deviations are shown in Table $V$ for all occurrences of $0.9<M_{I}<1.1$ in the Monte Carlo calculations. In general, it looks like $\gamma(r=a / 2) \sim 0.5 \mathrm{~m}^{2} / \mathrm{s}$ is required for ignition in CIT.

Ve will be comparing these values for $\gamma$ with those predicted by the Tang-Redi anomalous thermal transport model ${ }^{13-15}$ : trapped electron drift waves and the toroidal ion temperature gradient driven $\left(\eta_{i}\right)$ mode provide the scaling for $\chi$ in the "good confinement" zone $(1<q<2$ with $q$ being the 
MHD safety factor); the overall shape of $\chi(r)$ is fixed by a profile-consistency assumption.

Although not a true "first principles" theory, the Tang-Redi model has been able to reproduce experimental results for a large range of tokamak operating conditions. After being calibrated against TFTR ohmic discharges, ${ }^{13}$ it was used succesfully to predict performance in several tokamaks (TFTR. T-10, TEXT, Alcator-C, and ASDEX) in various regimes ${ }^{14}$ (ohmic heating, pellet injection, TFTR supershots, electron cyclotron resonance heating, and neutral beam heating).

A few minor additions must be made to the theory in order to apply it to Eq. (21). First, the Tang-Redi model differentiates between $\chi_{e}$ and $\chi_{i}$, the thermal diffusivities for the electron and ion channels, respectively. Likewise. there are distinct input powers, $P_{e}$ and $P_{i}$. Since we take $T_{e}=T_{i}$, we have only one $x(r)$,

$$
\chi(r)=\frac{\left(n_{e} X_{e}+n_{i} \chi_{i}\right)}{\left(n_{e}+n_{i}\right)}
$$

Furthermore, it is reasonable to set $P_{\varepsilon}=P_{i}=P_{\text {input }} / 2$. The Tang-Redi model assumes a circular cylindrical geometry, so we insert $\kappa$ anc $\delta$ factors into the Tang-Redi thermal diffusivity $\chi^{T R}$ to yield the original profileconsistent $T(r)$ when used in Eq. (21). This is equivalent to the generalization of the Tang model made by Jardin, et al. ${ }^{40}$ to noncircular geometry when their formula is evaluated for flux surfaces of constant $\kappa$ and $\delta$.

Taking the ratio of $\chi(r)$ computed from Eq. (21) to the value of $\chi^{T R}$ defined using Eq. (23), we find

$$
\frac{x}{\chi^{T R}}=\frac{a^{2}}{2 V} \frac{P_{\text {input }}}{\alpha_{T^{n}-0} T_{0}} \frac{\left[\left(1-\frac{r^{2}}{a^{2}}\right)^{1-\alpha_{T}} \exp \left(-\frac{2}{3} \alpha_{q} \frac{r^{2}}{a^{2}}\right)\right]}{\left(1-e^{-\alpha_{q}}\right)\left(\hat{\chi}_{e}+\frac{n_{i}}{n_{e}} \hat{\chi}_{i}\right)},
$$

where $\alpha_{q} \equiv q_{\text {eyl }}+0.5$. The term in brackets in the numerator contains all of the radial variation due to the deviation of our temperature profile from the Tang-Redi profile-consistent shape. The facturs in the denominator $\hat{\gamma}$ contain the essential microinstability scalings and the calibration factors. 
The dissipative and collisionless trapped electron modes give rise to

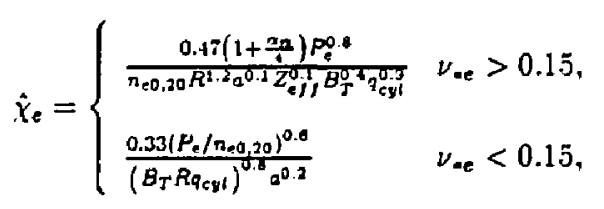

and

$$
\dot{\chi}_{i}^{T E M}= \begin{cases}0 & \nu_{e e}>0.15 \\ \frac{3}{2}\left(1+1 / \eta_{i}\right) \frac{L_{n}}{R} \hat{\chi}_{e} & \nu_{\text {ee }}<0.15,\end{cases}
$$

where $\nu_{e e}$ is the banana regime parameter and $P_{e}$ is in MW. Note that $\eta_{i} \equiv$ $d \ln T_{i} / d \ln n_{i}=\alpha_{T} / \alpha_{n}$ for parabolic profiles. We evaluate $L_{n} \equiv|d \ln n / d r|^{-1}$ at $r=a / 2$ to obtain $L_{n}=3 a / 4 \alpha_{n}$.

The toroidal ion temperature gradient driven $\left(\eta_{i}\right)$ mode yields

$$
\hat{\chi}_{i}^{\eta_{i}}= \begin{cases}0 & \eta_{i}<1 . \overline{5}, \\ \frac{0.5\left(P_{1} / n_{e 0.20}\right)^{0.8}}{\left(B_{T} R q_{c y l}\right)^{0.8} g^{0.2}} . & \eta_{i}>1.5,\end{cases}
$$

and $\dot{\chi}_{i}=\dot{\chi}_{i}^{T E M}+\dot{\chi}_{i}^{\eta_{i}}$.

Examination of our assumed ranges for $\alpha_{n}$ and $\alpha_{T}$ shows that the $\eta_{i}$ mode will always be off $\left(\dot{\chi}_{i}^{\eta_{i}}=0\right)$ for the peaked density profile cases, and will usually be on for the flat cases $\left(1<\eta_{i}<\infty\right)$. Furthermore, with $\nu_{\text {ee }}$ evaluated at $r=\alpha / 2$, we typically find $\nu_{* e} \sim 0.005-0.05$, so that only the collisionless trapped electron drift wave is active.

The values of $\chi / \chi^{T R}$ at $r=a / 2$ and their standard deviations are given in Table VI for all occurrences of $0.9<M_{I}<1.1$ in the Monte Carlo caiculations. The first thing we notice is that $\chi / \chi^{T R}$ for the peaked cases is more than twice as large as it is for the flat. The reason for this is just that $\hat{\chi}_{i}^{\eta_{i}}=0$ for the former. Secondly, it appears that for the most part $\chi / x^{T R}>1$. That is, our assumptions are more pessimistic than those of the Tang-Redi model. Pari of the reason for this is that only the collisionless trapped electron mode scaling is entering; the dissipative trapped electron mode is regarded as being less favorable. In the dppendix, we describe 1-1/2-D simulations with the Tang-Redi model indicating that CIT will ignite under circumstances analogous to the ones assumed here. 
Waltz, Dominguez. and Perkins have also developed a transport model based upon drift wave instabilities. ${ }^{6}$ They derived $\tau_{E}$ formulas explicitly for use in a $0-D$ power balance. When their model was applied to an earlier version of CIT, they found that ignition could be achieved only at very high densities $\left(\left\langle n_{e}\right\rangle \sim 10^{21} \mathrm{~m}^{-3}\right)$. However, they also argued that the empirical tokamak density limit [e.g.. Eqs. (19) and (20)] is likely to be brought on by excessive impurity radiation. By incorporating an approximate model for impurity line radiation into their calculations, they were able to show that with enough input power. CIT can burn through this empirical limit.

As a comparison, we insert their theoretical $\tau_{E}$ formula into our probability of ignition code. The results obtained are qualitatively similar to those discussed at length in Ref. 6. Hence, we wil] present no more detai] here except to describe the most favorable case. the $I_{p}=13$. $1 \mathrm{~A}$ configuration with flat density profiles. " $i$, hode continement as detined in Ref. 0 is assumed with no width to the distribution of $c_{r}$. We find in this case, $P\left(M_{I}>1\right)=5 \%, P(Q>10)=46 \%$. To reach ignition with all parameters evaluated at the means of the distributions (but still "H-mode confinement"). we would need $\left\langle n_{e}\right\rangle \simeq 8 \times 10^{20} \mathrm{~m}^{-3}$. The analysis of Waltz. Dominguez. and Perkins ${ }^{6}$ indicates that such densities can be readily achieved, as discussed . in the previous paragraph.

These results are more pessimistic than those found with the Tang-Redi model. This discrepancy may be the result of the dissimilar assumptions made in deriving the two theoretical models. ${ }^{6.13}$ We would be venturing too far beyond the scope of this paper to go into any greater detail on this point.

\section{Summary}

To summarize, we have deveioped a $0-D$ power balance code that uses a Monte Carlo algorithm to determine the probability of ignition, and have applied this code to six different configurations of CIT. The input for this code consists of probability distributions for the critical physics parameters in the 0 -D calculation. We have taken these distributions to be uniform (except for the Troyon coefficient. Fig. 6), and have used empirical knowledge to estimate reasonable ranges for the parameters in CIT. In addition, we have given statistical arguments suggesting that the L-mode energy confinement 
time in CIT will be essentially that given by the Faye-All-Complex expression [Eq. (17)] to within a standard deviation of $\sim 11 \%$.

With this basis, we have found that the probability of ignition is $\geq 6.5 \%$ for three of the six cases examined, and the probability of $Q>10$ is better than about $65 \%$ for five of the six cases (see Tables III and IV). In order for CIT to be operated in one of these five configurations, either the existing TFTR power supplies must be upgraded to allow CIT to achieve 11 MA of plasma current or some means of obtaining peaked density profiles must be developed. The sixth case is that of the 9 MA machine with flat density profiles. Even in this instance, the probability of reaching significant $Q$ is not small.

We have also found that peaking the density profile at fixed L-mode multiplier, $c_{T}$, to the extent described in Sec. IV.A increases the probability of ignition by about as much as raising the plasma current and toroidal field by $20 \%$.

The auxiliary heating requirements for CIT do not exceed 20 MW if ignited or very high $Q(Q \sim 50)$ is possible. In the most pessimistic cases, 20 MW of auxiliary heating would still be sufficient to allow interesting alpha physics experiments to be carried out $(Q \sim 5)$.

Finally, we have compared the level of confinement required at ignition with that predicted by the Tang-Redi theory-based transport model. ${ }^{13-15} \mathrm{We}$ find that our empirically based assumptions are slightly pessimistic relative to the theory results. On the other hand, the Waltz, Dominguez, and Perkins ${ }^{6}$ theory suggests that we are being overly optimistic. 


\section{Appendix}

We describe here 1-1/2-D simulations verifying the assertion made in Sec. VI that CIT will ignite with the Tang-Redi transport model. The BALDUR transport code is utilized for these simulations. More details on it, and how it has been employed previously in CIT simulations are given in Ref. 41 . The parameters for these runs have been chosen to match as closely as possible those of one point from the 11 MA peaked density profile Monte Carlo calculation. Namely, we take $\alpha_{n}=1.8, \alpha_{T}=1.3, Z_{e f f}=1.6 . g_{\beta}=2.2$. $h=3.6$, and $c_{T}=1.5$. The result of the $0 . \mathrm{D}$ calculation is $M_{l, \max }=1.0$. At this point, $n_{e 0}=9.1 \times 10^{20} \mathrm{~m}^{-3}$ and $T_{0}=16 \mathrm{keV}$.

The simulation is started with full fields and densities. A constant rolumeaveraged density $\left\langle n_{e}\right\rangle=3.4 \times 10^{20} \mathrm{~m}^{-3}$ is maintained by gas puffing. A simple particle diffusion model with diffusivity $D=0.5 \mathrm{~m}^{2} / \mathrm{s}$ is employed. We assume an anomalous pinch, $v \propto D r / a^{2}$. The constant of proportionality is adjusted to vield an effective profile peaking factor $\alpha_{n}^{e f f} \equiv n_{e} /\left(n_{e}\right\rangle-1 \simeq 1.3$; the constant we arrive at is 3.4 . Enough carbon impurity is added to bring $Z_{\text {eff }}$ up to 1.6. We assume that some mechanism for stabilizing sawtooth oscillations is available. This allows the density and temperature profiles to remain peaked at all times during the simulation. The plasma is initialized with a low temperature $\left(T_{0}=1 \mathrm{keV}\right)$ and then heated with $20 \mathrm{MW}$ of auxiliary power.

While we can force $\alpha_{n}$ and $Z_{\text {eff }}$ in the 1-1/2-D code to match the values used in the 0-D case, we cannot do so for $\alpha_{T}$. It is determined by the thermal transport model. The same can be said of $c_{\boldsymbol{r}}$. By choosing the same volumeaveraged density as the D-D code, the density limit specified by $h=3.6$ is adhered to. We must check the simulation results to verify that the $\beta$ limit is not exceeded.

The Tang-Redi thermal transport model is as described in Ref. 14 with two differences. The first is the addition of the $\kappa$ and $\delta$ factors mentioned in Sec. VI to generalize the theory to noncircular cross section. The second difference involves the transition between the collisionless and dissipative branches of the trapped electron mode. Normally, the crossover occurs when $\nu_{\text {we }}=0.15$ at the $q=1.5$ surface. To be consistent with the procedure of Sec. VI, we evaluate $\nu_{\text {*e }}$ at $r=a / 2$. Yote that this alteration affects only 
the transition, not the scaling of the thermal diffusivity.

In our first simulation, we set the critical $\eta_{i}, \eta_{i}^{\text {err }}$, to 1.5 as in Ref. 11 [see also Eq. (27)]. Two and one-half seconds into the simulation. the auxiliary heating is turned off. The plasma energy continues to rise at a rate of $d W_{\text {tot }} / d t \simeq 3 \mathrm{MW}$ [see Fig. 11(a)]. So, this case is ignited. but not by much. In fact, the ignition margin at three seconds into the run is $\boldsymbol{M}_{J} \simeq 1.0$.

$\lambda$ is clear from Fig. 11(a), the ion temperature profile is very peaked despite the presence of $\eta_{i}$ mode transport. However. for all of the peaked density profile cases in our $0-\mathrm{D}$ calculations $\eta_{i}<1.5$, and there is no $\eta_{i}$ mode transport. Our second simulation forces this to be the case in the 1-1/2-D code as well by setting $\eta_{i}^{\text {rrtt }}=4.5$. In this instance, only one second of 20 WW auxiliary heating is required to bring the plasma to ignition. At $t=1.5$ s. $M_{I}=1 . T$. The ion temperature profile continues to peak until $\eta_{i}>\eta_{1}^{\text {irte }}$. The $\beta$ limit used in the $0-D$ code, $\beta_{\max }=2.2 I_{p} / a B_{T}$, is exceeded only near the end of this simulation $(t \simeq 1.8)$.

These simulations are more optimistic than the 1-1/2-D Tang-Redi model simulation described in Ref, 41 . The primary reasons for this are: 1) by assuming we can stabilize the sawtooth oscillations, peaked density and temperature profiles can be maintained at all times, and 2) the present simulations are always in the collisionless regime for trapped electron modes. The simulation described in Ref. 41 had a thermal diffusivity with contributions from both the collisionless and the (less favorable) dissipative branches.

\section{Acknowledgments}

The authors would like to acknowledge the many valuable contributions to this project made by the CIT team. In addition, we wish to thank Dr. S. M. Kaye for providing access to the L-mode database. This work was supported by U.S. DOE Contract No. DE-AC02-76-CHO-3073. 


\section{References}

'W. A. HOULBERG. S. E. ATTENBERGER, and L. M. HIVELY, "Contour Analysis of Fusion Reactor Plasma Performance." Vucl. Fusion. 22. 935 (1982).

${ }^{2}$ N. A. LCKAN and J. SHEFFIELD, "A Simple Procedure for Establishing Ignition Conditions in Tokamaks," Tokamak Startup, p. 45. H. KNOEPFEL, Ed., Plenum Press. New York (1986).

${ }^{3}$ Y. C. SUN, D. E. POST. G. BATEMAN. and D. STOTLER. "The Performance of CIT and ITER Lnder Various Scaling Laws." Bull. Am. Phys. Soc., 33. 1971 (1988).

${ }^{4} 0$. MITARAI, A. HIROSE, and H. M. SKARSGARD, -Generalized Ignition Contour Map and Scaling Law Requirement for Reaching Ignition in a Tokamak Reactor," Vucl. Fusion, 28, 2141 (1988).

${ }^{5} \mathrm{~N}$. A. UCKAN, Relative Merits of Size, Field, and Current on Ignited Tokamak Performance," Fusion Technol, 14, 299 (1988).

${ }^{6}$ R. E. WALTZ, R. R. DOMINGUEZ, and F. W. PERKINS, "Drift Wave Model Tokamak Ignition Projections with a Zero-Dimensional Transport Code," Nucl. Fusion, 29, 351 (1989).

'R. E. WALTZ, R. R. DOMINGUEZ, F. W. PERKINS, J. C. WILEY, W. H. MINER, D. W. ROSS, P. W. TERRY, P. M. VALANJU, P. H. DIAMOND, and J. SHEFFIELD, "Theoretical Models for Tokamak Ignition Projections," Proc. 12th Int. Conf. Plasma Physics and Controlled Nuclear Fusion, Nice, France, November 1988, paper IAEA-CN-50/G-2-3, International Atomic Energy Agency.

${ }^{8}$ S. K. HO and L. J. PERKINS, "Probability of Ignition: A Better Approach than Ignition Margin," Nucl. Fusion, 29, 81 (1989).

${ }^{9}$ S. M. KAYE, Princeton Plasma Physics Laboratory, Private Communication (1986).

${ }^{10}$ "Crystal Ball - A Forecasting and Risk Management Program for the Macintosh," Market Engineering Corporation (Denver, Colorado. 1988). 
${ }^{11}$ R. R. PARKER et al.. "CIT Physics and Engineering Basis." Proc. I:th Int. Conf. Plasma Physics and Controlled Nuclear Fusion. Nice. France. November 1988, paper [AEA-CN-50/G-2-1, International Atomic Energy Agency; see also J. A. SCHMIDT and H. P. FURTH. "Compact Ignition Tokamak Conceptual Design Report." A-860606-P-01. Princeton Plasma Physics Laboratory (June 1986).

${ }^{12}$ F. WAGNER, G. BECKER. K. BEHRINGER, D. CAMPBELL, A. EBER. HAGEN, et al., "Regime of Improved Confinement and High Beta in Neutral-Beam-Heated Divertor Discharges of the ASDEX Tokamak," Phys. Rev. Lett., 49. 1408 (1982).

${ }^{13}$ M. H. REDI, W. M. TANG, P. C. EFTHIMION, D. R. MIKKELSEN, and G. L. SCHMIDT, "Transport Simulations of Ohmic TFTR Experiments with Microinstability Based. Profile Consistent Models for Electron and Ion Thermal Transport," Nuci Fusion, 27, 2001 (1987).

${ }^{14}$ M. H. REDI, W. M. TANG, N. BRETZ, P. C. EFTHIMION, M. C. ZARNSTORFF, S. J. ZWEBEN. and D. L. BROWER, “Transport Simulations of Experiments on T-10, TEXT, and TFTR Using Microinstability Based Transport Models,' Bull. Am. Phys. Soc., 33, 2094 (1988).

${ }^{15}$ W. M. TANG, N. L. BRETZ, T. S. HAHM, W. W. LEE, F. W. PERKINS. M. H. REDI, G. REWOLDT, M. C. ZARNSTORFF, S. J. ZWEBEN. R. D. SYDORA, J. M. DAWSON, V. K. DECYK, H. NAITOU, T. KAMIMURA, and Y. ABE, "Theoretical Studies of Enhanced Confinement Properties in Tokamaks," Proc. 12th Int. Conf. Plasma Physics and Controlled Nuclear Fusion, Nice, France, November 1988, paper IAEACN-50/D-3-3, International Atomic Energy Agency.

${ }^{16}$ L. M. HIVELY, "Convenient Computational Forms for Maxwellian Reactivities," Nucl. Fusion, 17, 873 (197i).

${ }^{17 K}$. B. AXON, W. H. M. CLARK, J. G. CORDEY, M. COX, S. J. FIELDING, et al., "Beam-Driven Currents, Power Balances and Density Limits with Neutral Injection into DITE," Proc. 8th Int. Conf. Plasma Physics and Controlled Nuclear Fusion, Brussels, Belgium, July 1-10. 1980. p. 413, IAEA-CN-38/N-4, International Atomic Energy Agency. 
${ }^{27}$ KURT S. RIEDEL, "Advanced Statistics for Tokamak Transport Colinearity and Tokamak to Tokamak Variation," New York Liniversity. Courant Institute of Mathematical Sciences Report DOE/ER/53223-98 (March 1989).

${ }^{28}$ R. J. GOLDSTON, "Energy Confinement Scaling in Tokamaks: Some [mplications of Recent Experiments with Ohmic and Strong Auxiliary Heating," Plasma Phys. Controlled Fusion, 26, 87 (1984).

${ }^{29}$ Contributions to the IAEA Technical Meeting on H-mode Physics. Gut Ising, FRG, March 20-22. 1989, International Atomic Energy Agency.

${ }^{30}$ D. POST, Princeton Plasma Physics Laboratory, Private Communication (1989).

${ }^{31}$ S. M. KAYE, Princeton Plasma Physics Laboratory, Private Communication (1989).

${ }^{32}$ E. J. STRAIT, L. L. LAO, T. S. TAYLOR. M. S. CHU, J. K. LEE. A. D. TURNBULL, et al., "Stability of High Beta Discharges in the DIII-D Tokamak," Proc. 12th Int. Conf. Plasma Physics and Controlled Nuclear Fusion, Nice, France, November 1988, paper IAEA-CN-50/A-2-1, International Atomic Energy Agency.

${ }^{33}$ D. POST, W. HOULBERG, G. BATEMAN et al., "Physics Aspects of the Compact Ignition Tokamak," Physica Scripta, T16, 89 (1987).

${ }^{34}$ T. PETRIE, N. BROOKS, A. KELLMAN, M. MAHDAVI, T. OSBORNE. D. CONTENT, and D. HILL, "High-Density Limits in Ohmic- and BeamHeated Plasmas in DIII-D," Bull. Am. Phys. Soc., 33, 1965 (1988).

${ }^{35}$ D. J. CAMPBELL, P. A. DUPERREX, A. W. EDWARDS, R. D. GILL. C. W. GOWERS, et al.. "Sawteeth and Disruptions in JET," Proc. 11th Int. Conf. Plasma Physics and Controlled Nuclear Fusion. Kyoto. Japan, November 13-20, 1986, p. 433, IAEA-CN-47/A-VIJ-5, International Atomic Energy Agency.

${ }^{36}$ D. P. STOTLER and N. POMPHREY, "Pulse Length Assessment of Compact Ignition Tokamak Designs," PPPL-2630, Princeton Plasma Physics Laboratory (1989); see also Fusion Technol. (to be published). 
${ }^{37}$ E. Ibrahim, University of California at Los Angeles. Private Communication (1989).

${ }^{38} \mathrm{~B}$. COPPI and THE IGNITOR PROGRAM GROC'P, "Characteristics and Expected Performance of the Ignitor-U Experiment," Proc. 1:th Int. Conf. Plasme Physics and Controlled Vuclear Fusion. Vice. France, November 1988, paper IAEA-CN-50/G-2-2. International Atomic Energy Agency.

${ }^{39}$ K. H. BURRELL, S. L. ALLEN, G. BRAMSON, N. H. BROOKS, R. WV. CALLIS, et al., "Energy Confinement in Auxiliary-Heated Divertor and Limiter Discharges in the DIII-D Tokamak," Proc. I2th Int. Conf. Plasma Physics and Controlled Nuclear Ftssion. Nice, France, November 1988. paper IAEA-CN-50/A-3-4-2, International Atomic Energy Agency.

${ }^{40}$ S. C. JARDIN, J, DE LUCIA, M. OKABAYASHI, N. POMPHREY, M, REUSCH, S. KAYE, and H. TAKAHASHI, "Post-Disruptive Plasma Loss in the Princeton Beta Experiment (PBX), Nucl. Fusion, 27, 569 (1987).

${ }^{41}$ D. P. STOTLER and GLENN BATEMAN, "Time-Dependent Simulations of a Compact Ignition Tokamak," Fusion Technol., 15, 12 (1989). 


\begin{tabular}{|l|c|c|}
\hline Parameter & CIT & Experiment \\
\hline ion collisionality, $\nu_{-i}$ & $10^{-2}$ & PLT \\
electron collisionality, $\nu_{e}$ & $10^{-2}$ & TFTR, JET \\
magnetic Reynolds number, $S$ & $10^{9}$ & TFTR, JET \\
$\beta$ & $5 \%$ & DIII-D \\
relative ion Larmor radius, $\rho_{i} / a$ & $10^{-3}$ & TFTR, JET \\
relative neutral mean-free path, $\lambda_{m j \rho}^{\circ} / a$ & $10^{-2}$ & Alcator C \\
\hline
\end{tabular}

Table I: Dimensionless parameters used to mark theoretical boundaries. the order of magnitude expected in CIT, and recent experiments that have achieved similar values.

\begin{tabular}{|c|c|}
\hline Parameter & Range \\
\hline$c_{\tau}$ & $1-2.5$ \\
$\alpha_{n}^{\text {flat }}$ & $0-0.75$ \\
$\alpha_{n}^{\text {peaked }}$ & $1.5-3$ \\
$\alpha_{T}$ & $0.75-1.75$ \\
$\eta_{o}$ & 0.95 \\
$Z_{\text {eff }}$ & $1.3-2$ \\
$g_{\beta}^{\text {flat }}$ & $2-3.5$ \\
$g_{\beta}^{\text {peaked }}$ & $1.5-3$ \\
$h$ & $1.5-4$ \\
\hline
\end{tabular}

Table II: Ranges of critical physics parameters assumed for probability distributions input to the Monte Carlo calculation. All distributions are uniform except that of $g_{a} ;$ it is as in Fig. 6.

\begin{tabular}{|c|c|c|}
\hline$I_{p}$ & flat & peaked \\
\hline $9 \mathrm{MA}$ & $9 \%$ & $40 \%$ \\
$11 \mathrm{MA}$ & $42 \%$ & $68 \%$ \\
$13 \mathrm{MA}$ & $65 \%$ & $88 \%$ \\
\hline
\end{tabular}

Table III: Probability of achieving $M_{I}>1$. 


\begin{tabular}{|c|c|c|}
\hline$I_{p}$ & flat & peaked \\
\hline $9 \mathrm{MA}$ & $39 \%$ & $66 \%$ \\
$11 \mathrm{MA}$ & $67 \%$ & $89 \%$ \\
$13 \mathrm{MA}$ & $86 \%$ & $99.5 \%$ \\
\hline
\end{tabular}

Table IV: Probability of achieving $Q>10$.

\begin{tabular}{|c|c|c|}
\hline$I_{p}$ & flat & peaked \\
\hline 9 MA & $0.27 \pm 0.06$ & $0.38 \pm 0.11$ \\
1I MA & $0.35 \pm 0.11$ & $0.53 \pm 0.15$ \\
13 MA & $0.44 \pm 0.14$ & $0.67 \pm 0.22$ \\
\hline
\end{tabular}

Table V: Mean value of $\chi(r=a / 2)$ and its standard deviation in $\mathrm{m}^{2} / \mathrm{s}$ for all occurrences of $0.9<M_{I}<1.1$ in the Monte Carlo calculations.

\begin{tabular}{|c|c|c|}
\hline$I_{p}$ & flat & peaked \\
\hline $9 \mathrm{MA}$ & $0.82 \pm 0.40$ & $1.9 \pm 0.33$ \\
$11 \mathrm{MA}$ & $1.0 \pm 0.56$ & $2.4 \pm 0.44$ \\
$13 \mathrm{MA}$ & $1.2 \pm 0.67$ & $3.0 \pm 0.58$ \\
\hline
\end{tabular}

Table VI: Mean value of $\chi / \chi^{T R}$ at $r=a / 2$ and its standard deviation for all occurrences of $0.9<M_{l}<1.1$ in the Monte Carlo calculations. 


\section{Figures}

FIG. 1. Experimental L-mode confinement time $\tau_{E}^{E X P}$ for the entire database plotted against a regression fit to Eq. (14), $\tau_{E, L}^{M, E}$, using just the "medium" and "big" portions of the database.

FIG. 2. Frequency distribution of $\ln \left(\tau_{E}^{E X P} / \tau_{E . L}^{M B}\right)$. Only the "medium" and "big" portions of the database are used. The best-fit gaussian curve is drawn for comparison.

FIG. 3. Plot of 1000 coefficients satisfying Eq. (16) for the "medium" and "big" portions of the L-mode database at the $67 \%$ confidence level; $b_{\text {; }}$ $\left(b_{3}\right)$ is the exponent on $a(R)$ in Eq. (14).

FIG. 4. Plot of 1000 coefficients satisfying Eq. (16) for the "medium" and "big" portions of the L-mode database at the $67 \%$ confidence level: $b_{4}$ $\left(b_{7}+b_{8}\right)$ is the exponent on $I_{p}$ (size) in Eq. (14).

FIG. 5. Value of $\tau_{E, L}$ obtained for CIT assuming $R=2.1 \mathrm{~m}, a=0.65 \mathrm{~m}$, $\kappa=2, B_{T}=10 \mathrm{~T}, I_{p}=11 \mathrm{MA}, \bar{n}_{e}=5 \times 10^{20} \mathrm{~m}^{-3}, \bar{A}_{i}=2.5$, and $P_{\text {input }}=100 \mathrm{MW}$ for 1000 sets of coefficients satisfying $F$.q. (1i) (using the combined "medium" and "big" portions of the database) at the $67 \%$ confidence level.

FIG. 6. Probability distributions assumed for the Troyon coefficient $g_{\mathcal{\beta}}$ for (a) ffat and (b) peaked density profiles.

FIG. 7. Probability distribution of $M_{I}(\mathrm{a})$ and the integral of the probability distribution above a given $M_{I}$ (b) for the cases with the flat density profiles.

FIG. 8. Probability distribution of $M_{I}$ (a) and the integral of the probability distribution above a given $M_{I}$ (b) for the cases with the peaked density profiles.

FIG. 9. Contours of constant auxiliary power in MW (solid lines) and $Q$ (dashed lines) in $\left\langle n_{e}\right\rangle$ and $\langle T\rangle$ space for the $9 \mathrm{MA}$ design with flat density profiles. All critical parameters are evaluated at the means of their respective distribution functions. The density and $\beta$ limits are indicated. 
FIG. 19. Contours of constant auxiliary power in MW (solid lines) and $Q$ (dashed lines) in $\left\langle n_{e}\right\rangle$ and $\langle T\rangle$ space for the 11 .MA design with flat density profiles. All critical parameters are evaluated at the means of their respective distribution functions, except that $c_{r}$ is reduced to 1.46 . The density and $\beta$ limits are indicated.

FIG. 11. Ion temperature as a iunction of radial half-width (half of the midplane width of a flux surface) and time in the 1-1/2-D simulations. The critical $\eta_{i}$ is set at 1.5 (a) and 4.5 (b). 


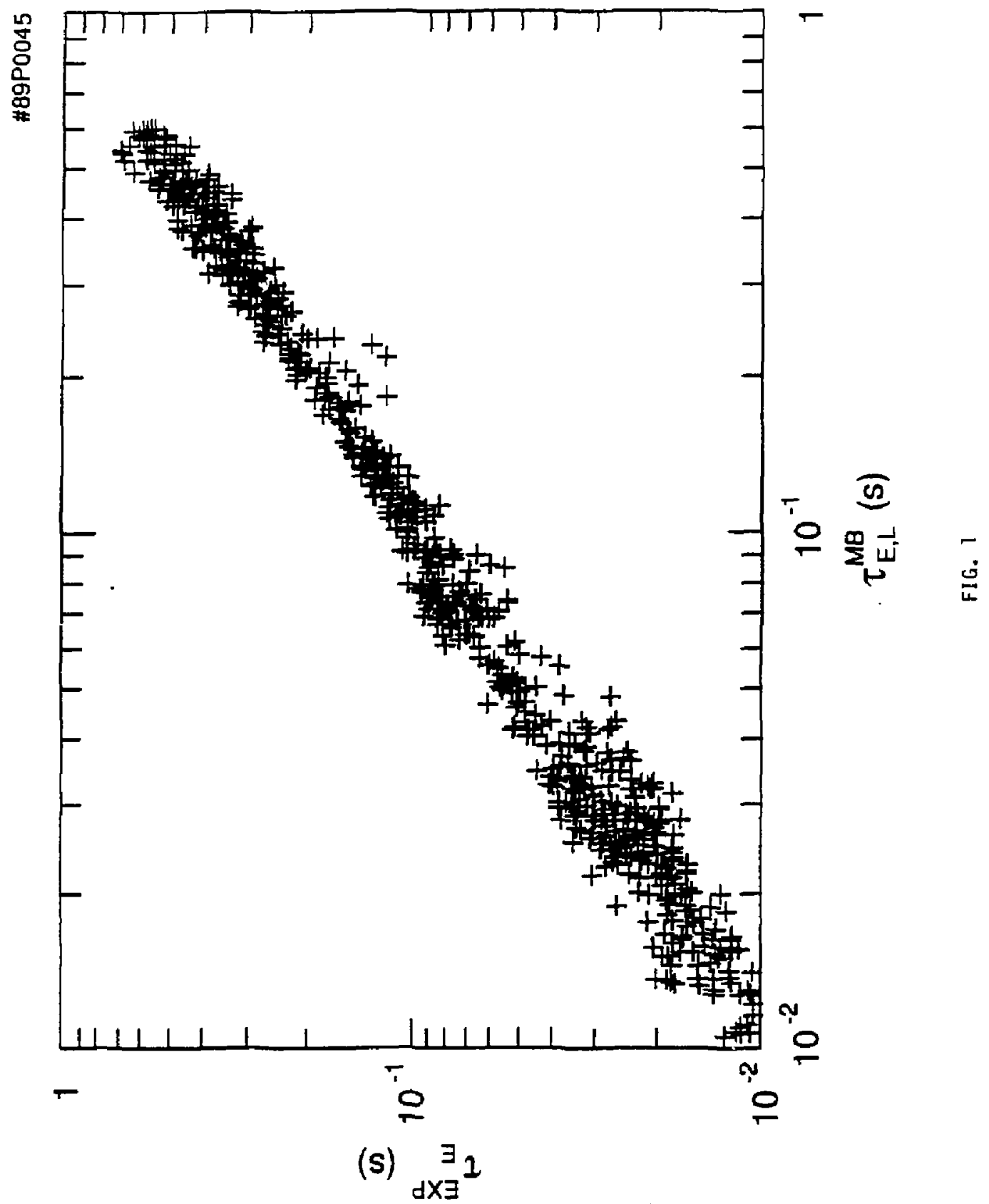


FREQUENCY

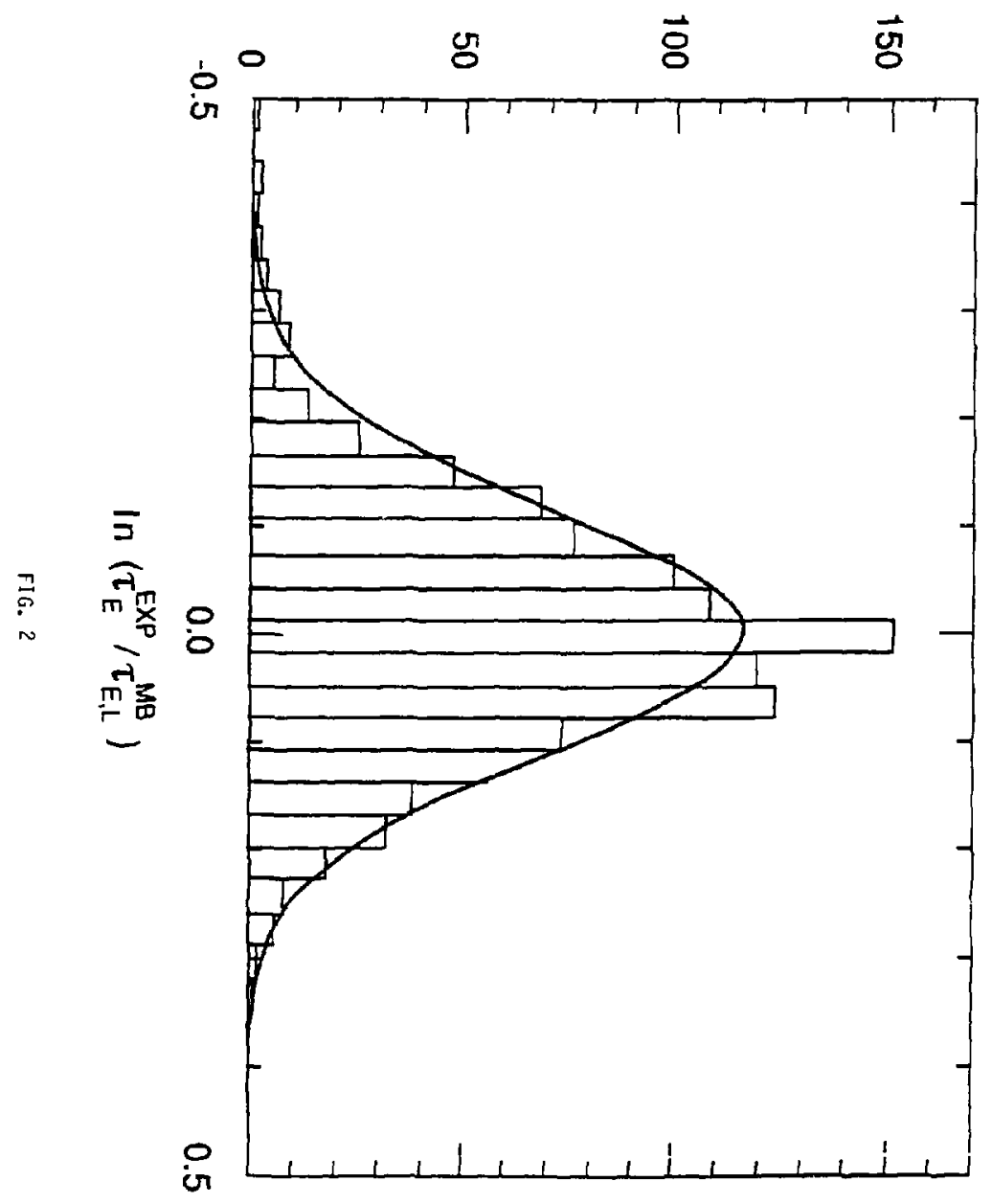

\begin{tabular}{l}
7 \\
0 \\
0 \\
0 \\
0 \\
0 \\
\hline
\end{tabular} 
\#89P0046

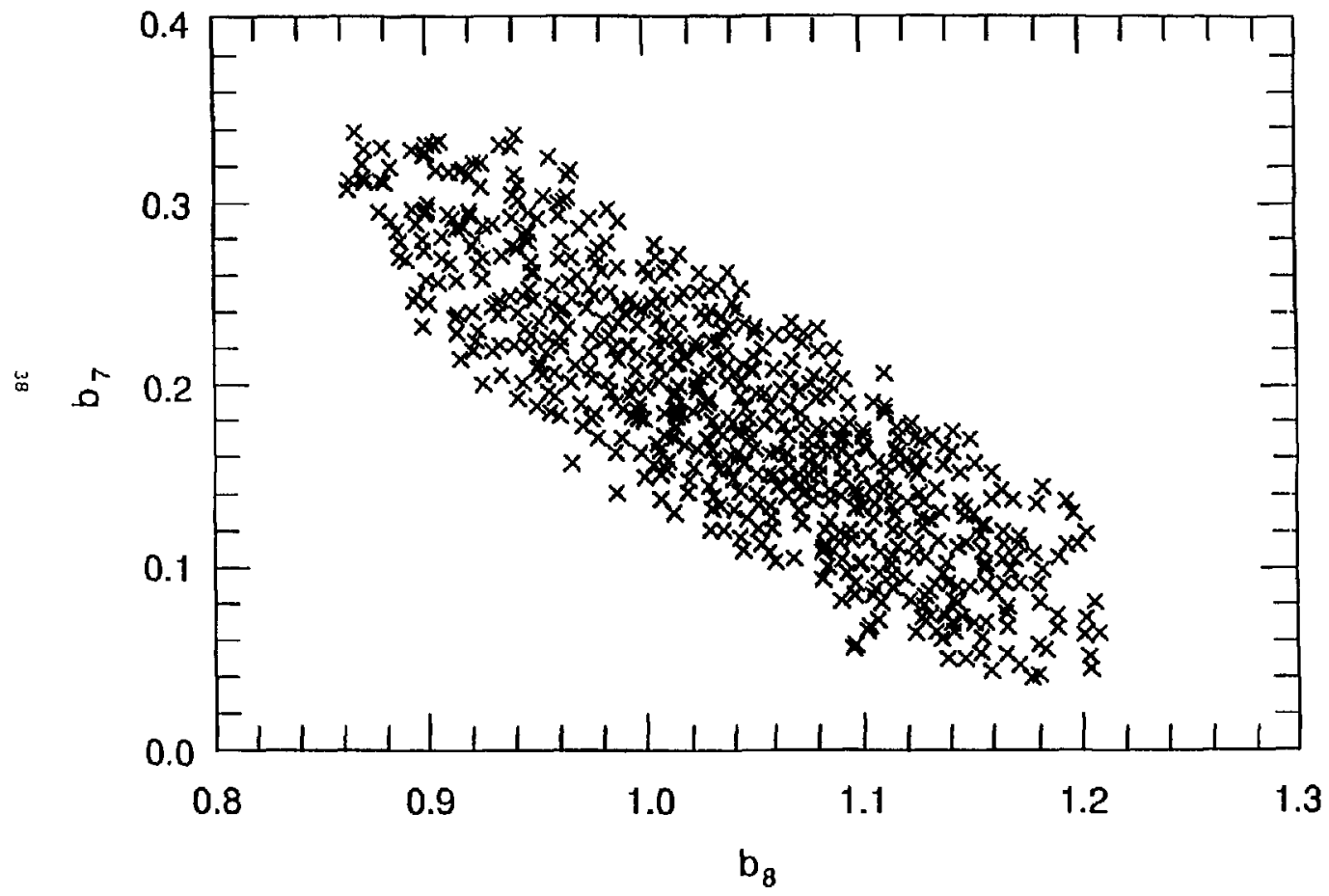

FIG. 3 


\section{\#89P0047}

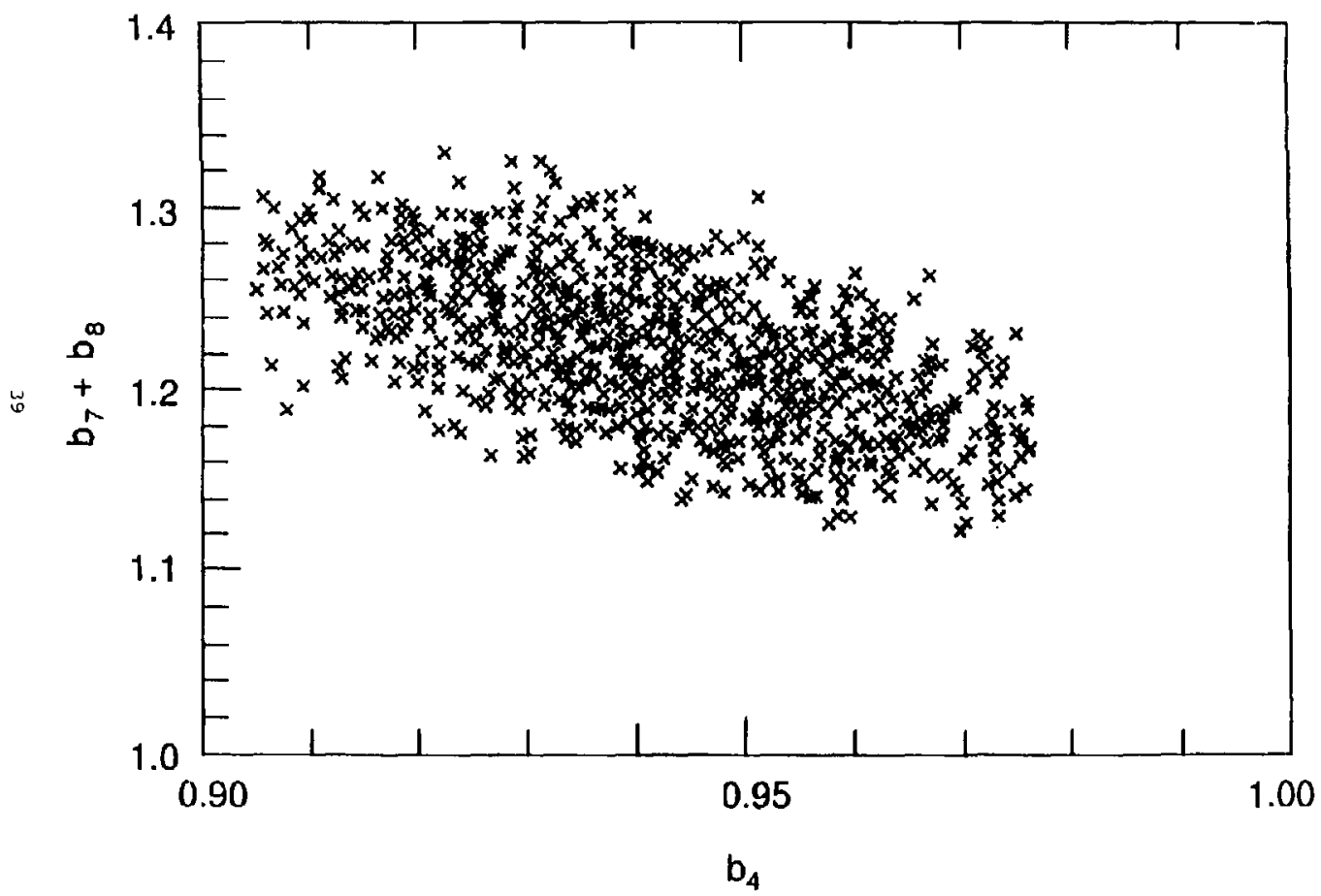

F1G. 4 


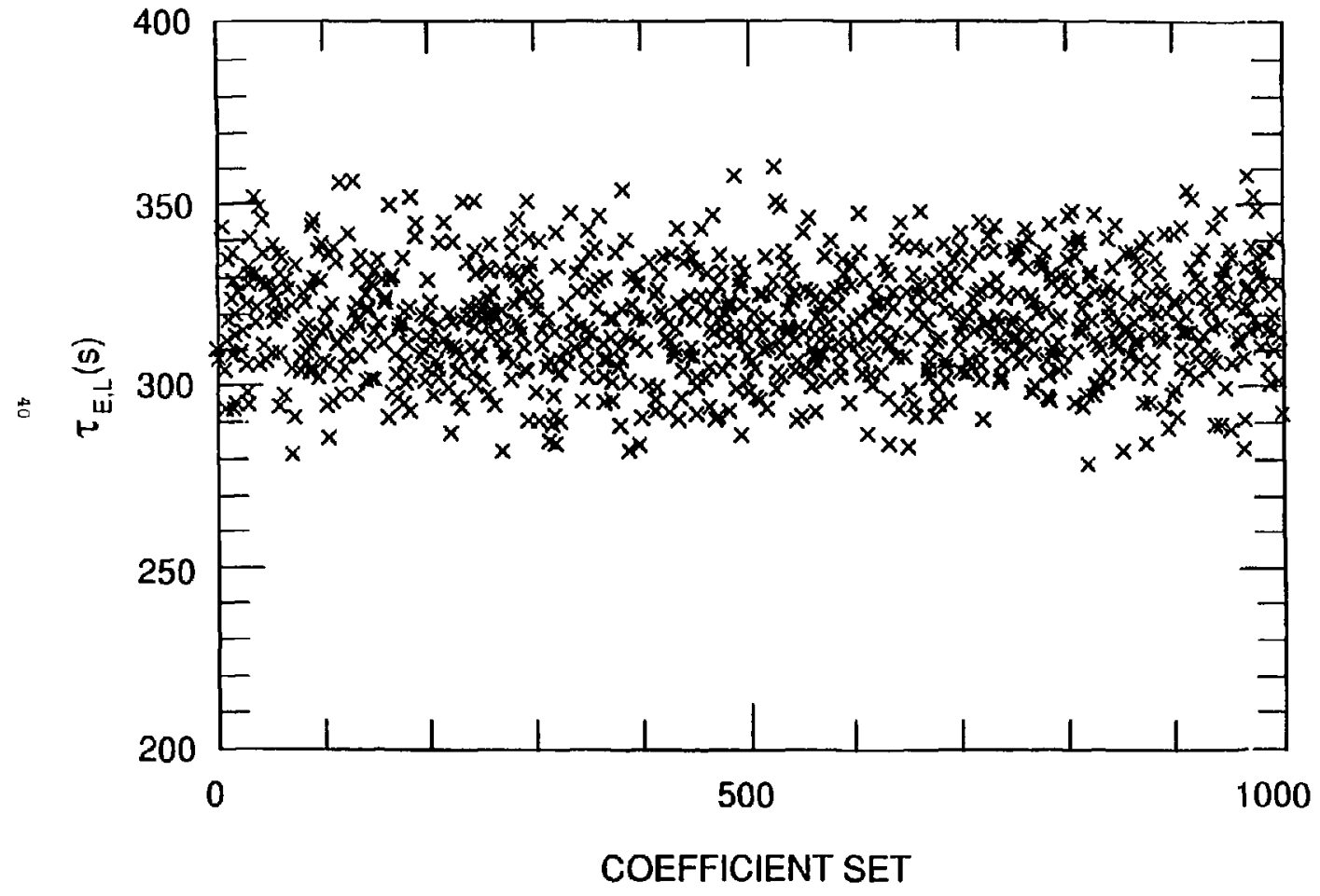

FIG. 5 

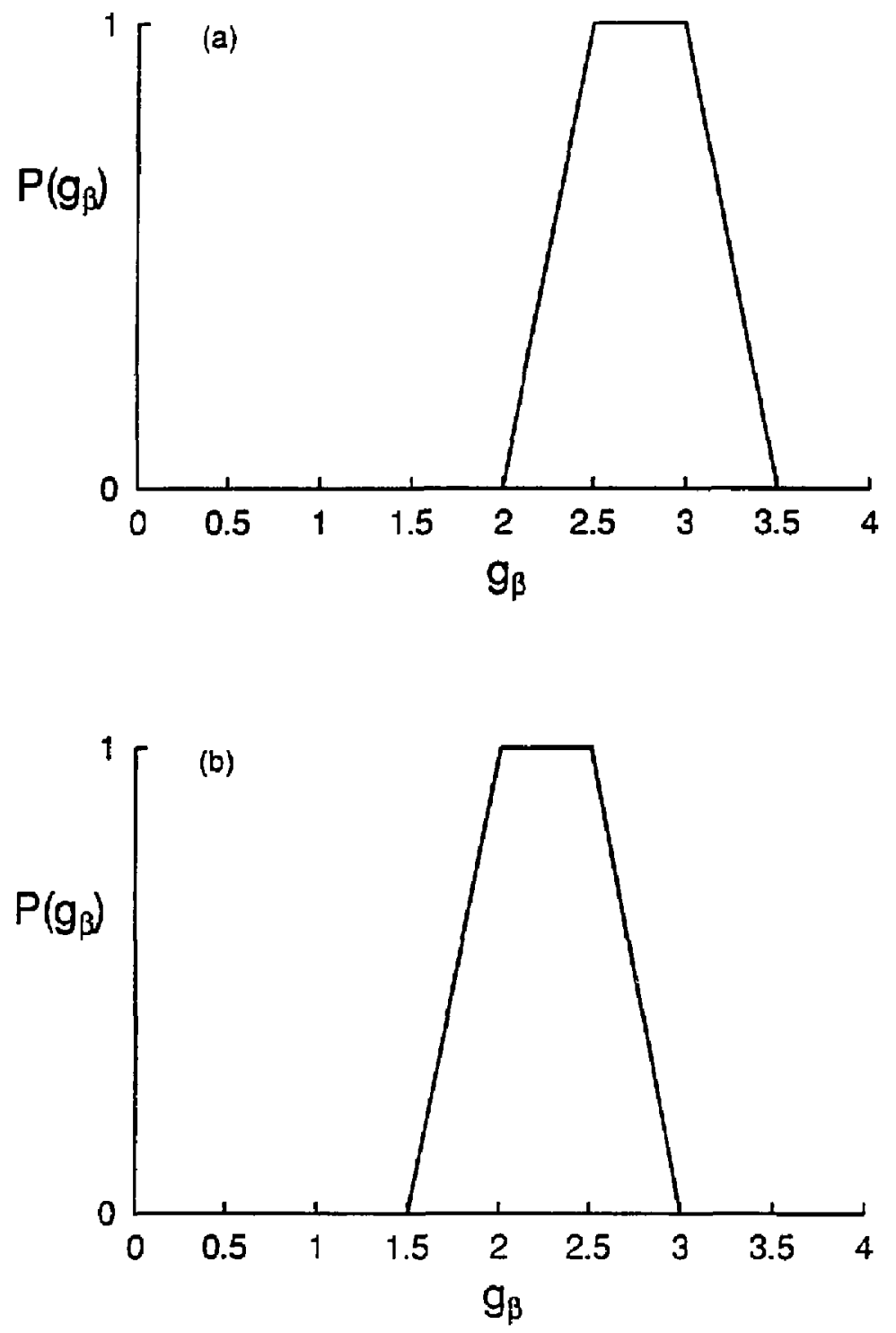

FIG. 6 

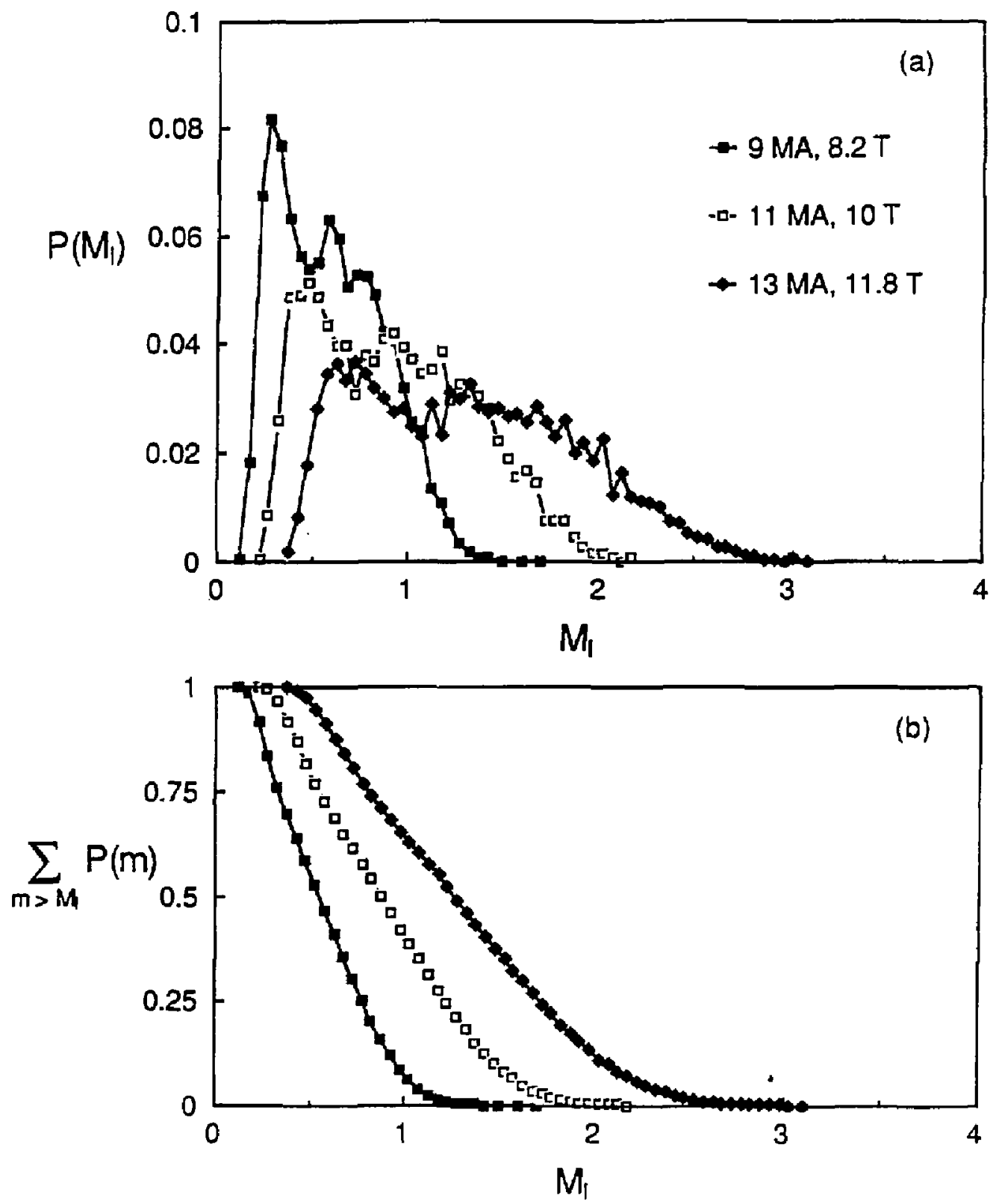

EIG. 7 

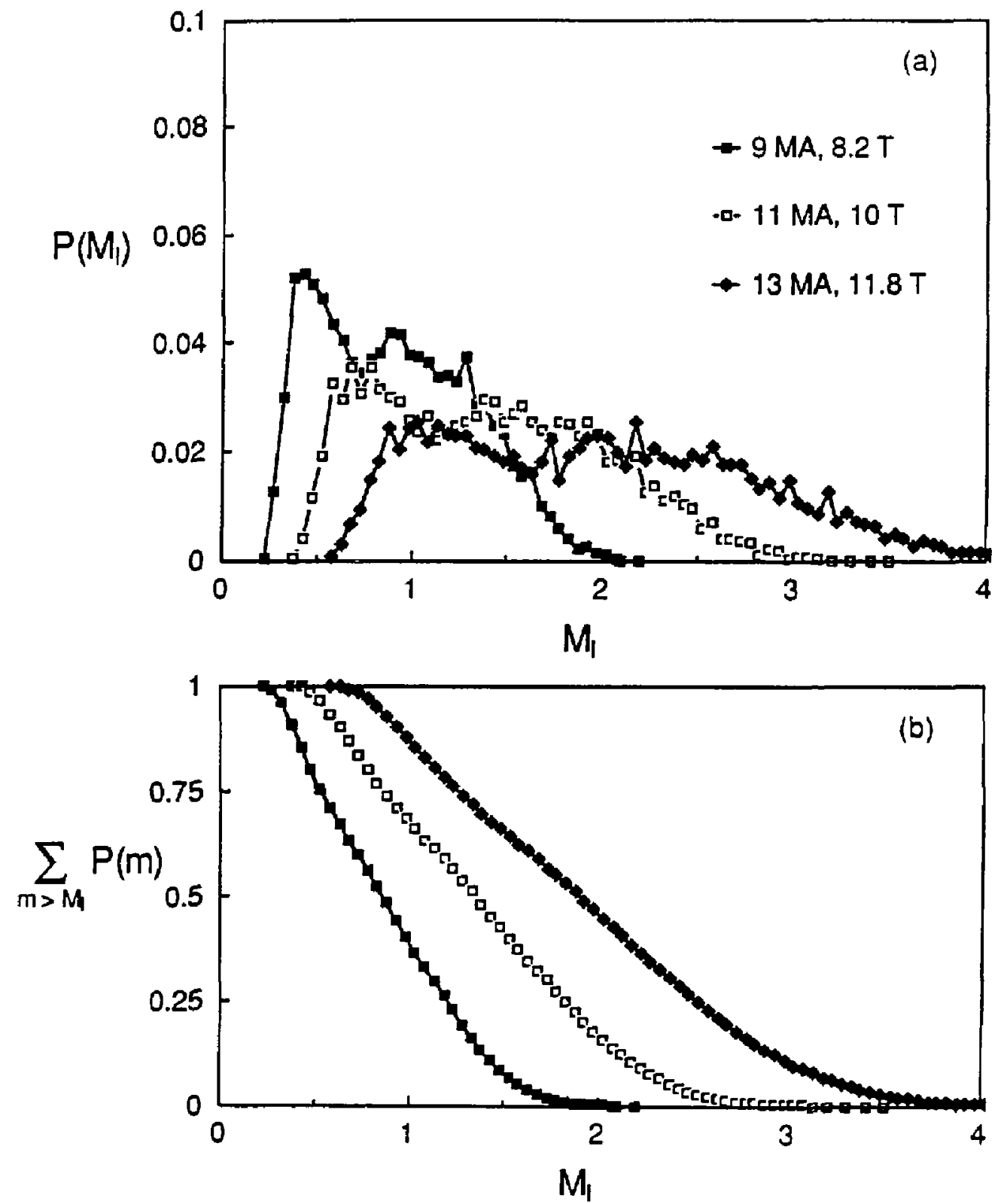

FIG. 8 


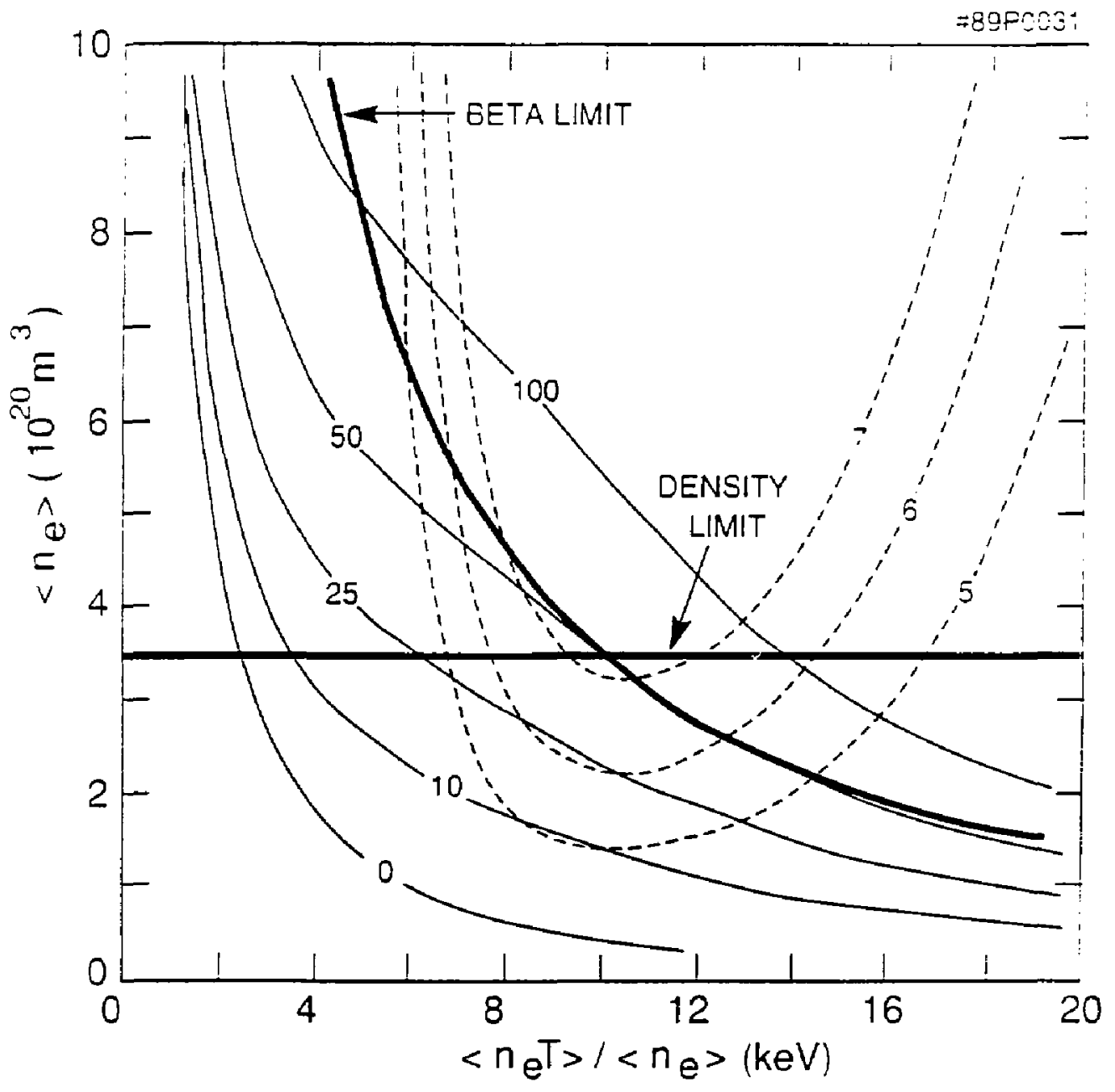

EIG. 9 


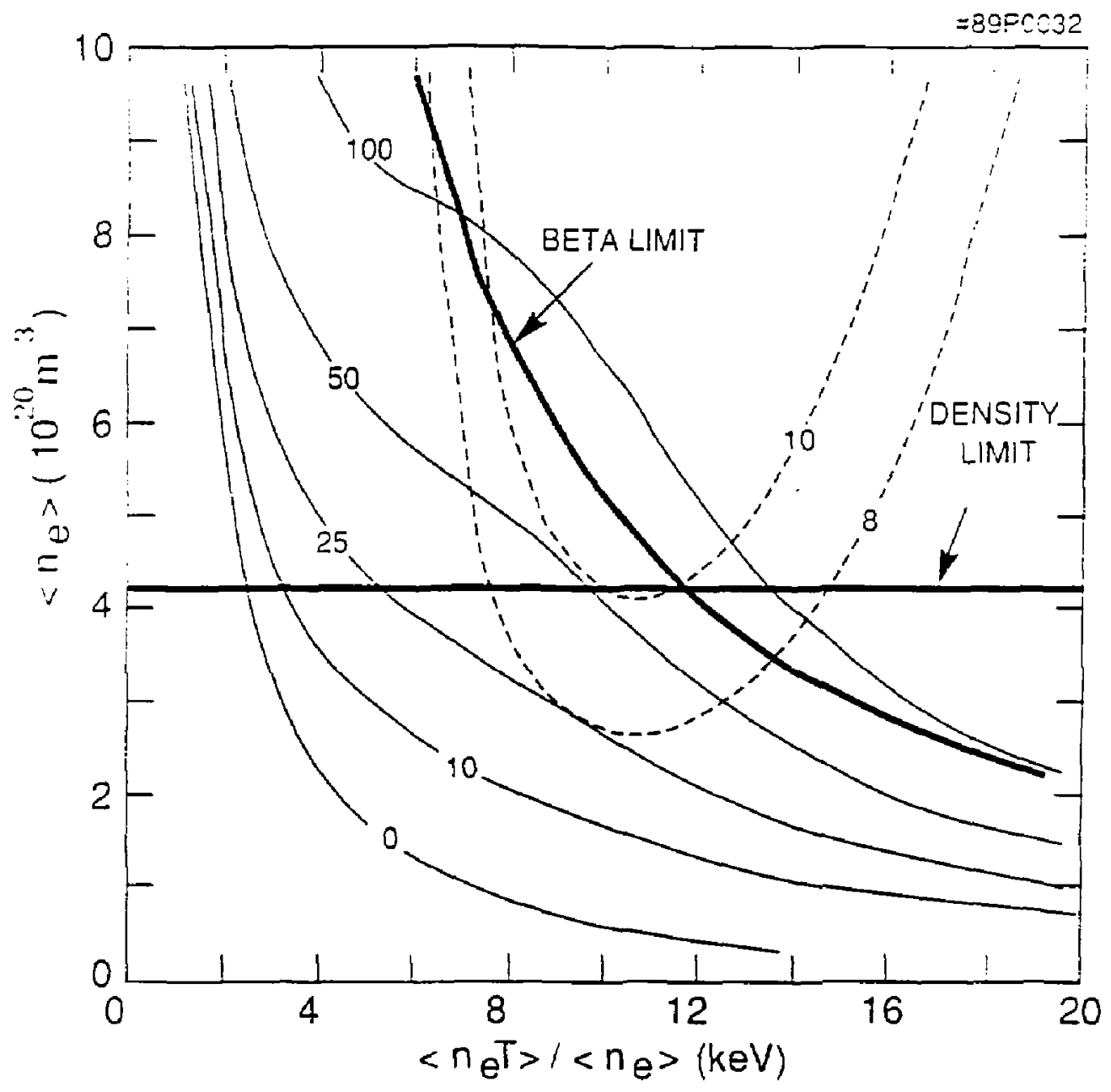

FIG. 10 

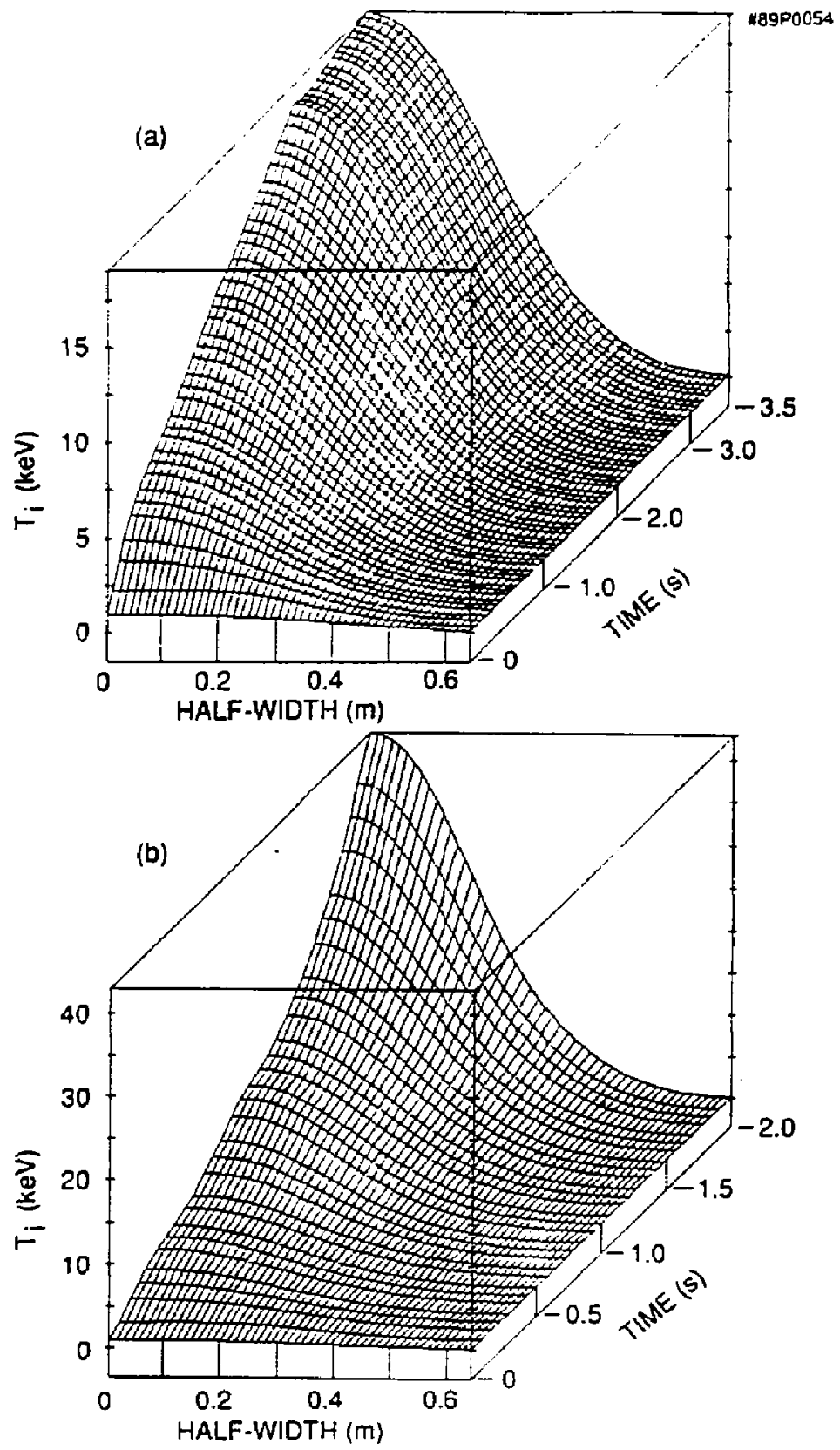

FIG. 11 
Dr. Frank J. Peolon 1, Univ of Wol longong, australia Prof, M,H, Bremnen, Univ Sydney, austanalia PI ased Rasearch Lob., Australi an Mat. Univ., AUSTRAL: Prot. I.R, Jones, Flinders Univ.., Austaklih Prof. F. Cop, Inst Theo Phys, AustRia

Prof. M. Hoindler, Instut fur Theoretlache Physik, AUSTRIA M. Goosens, Astrongisen InstituUt, BELGIUM Ecole Royule milltalre, La de Phys PIasas, Belgium Comissian-Europeen, $D_{9}-x_{1} I$ Fuslan Prog, BELGILM Prot. R. Boucique, Rijksuniversiteit Gant, Beigium Dr. P.H. Sakanaka, Instituto Fisice, BRMzIL Instituto De Pasquisas Espaciasi-INPE, BRAZIL Documents Office. Atcoic Energy af Canada Limited, CAmoA Dr. M.P. Bechynskl, we Technologies, Ine., CAMADA Dr. H.M. Skersgard, University of Saskatehaman, CAMADA Dr. H. Barnard, University of British Coluabia, caman Prof. J. Teichmann, Univ. of Montreal, CANDDA Prof. S.R. Sremivasen, Univarsity of Calgary, Canada Prof. Tudor W, Johnstoo, I Hes-Energie, CAwOA Or. Bolton, Centre canadien de fusion angnétique, cakada Or. C.R. Jams, Univ. of Aloarta, CNMOA

Dr. Pater Lukac, Komenskeno Univ, CZECHOSLOVNKIA

The Librarian, Culhe Luboratory, EMGLND

The Librarian, Rutherford Applaton Laboratory, EKGLND Mrs. S.A. Hutchinson, JET Library, ENCAANO C. Houttat, Lab. de Physlaue das MIl ieux lonisés, France J. Radet, CEN/CADARAGHE - Bat 506, FRANCE

Ms. C. Riani, Librarion, Univ. of loannina, GREECE

Or. Ton Mul, Acadeay Bibliographic Ser., howg kONG

Preprint Liorary, Hungarlan Acadary of Sciences, HUNGaRY Or. B. Das Gupta, Sohe inst of Nuel. Phys., INOIA Or. P. Kan, Institute for Plassa Researeh, INDIA Or. Philip Rosunau, Israel Inst, of Tech, ISRAEL Librarian, 1nt'I Ctr Theo Phys, ITALY

Prof. G. Rostagni, Istituto Gas lonizzati Dal CNR, ITALY Miss Clelio De Palo, Assoc ELRATOM-ENEA, ITALY Or. G. Grosso. Istituto di Fisica del plasma, HTALY Dr. H. Yamato. Tosniba Ras a Dev, Japan
Prof. 1. Kowakemi, Atomic Enorgy Ras. Instituto, JAPAN

Prot. KYoji Nishikawa, Univ of Hiroshima, JAPAN

Director, Oedt, Large Tohanak Res. JAERI, JAPAN

Prot, Sotoshi Itoh, Kyushu University, JAPAN

Researen Into Conter, Magoyo University, JAPAN

Prof. 5. Tonaka, Kyoto Iniversity, JAPAN

Library, Kyoto University, JAPMN

Prot. Nobuyuki Inoue, University of Tokyo, JAPAN

5. Mor i, JAERI, JAPAH

H. Jeong, Librarian, Kores Adyanced Energy Ras Inst, KCREA Prof. D.1. Choi. The Kores Ady. Ingt of Sei \& Jech, KOREA Prof. B.S. LIley, University of Waikato, NEW ZEALAND Institute of Plasan Physics, PEOPLE'S REPLiblic of CHINA Librarian, Institute of Phys., PEOPLE's REPUBLIC OF CHINA Library, Tsing twa University, PEOPLE'S REPUBLIC Of CHINA 2. Li, Southwast Inst. Physics, PECPLE'S REPUBLIC OF CHINA Prof, J.A.C. Cabral, Ingt Superior Tocnico, PORTUGAL Dr. Oetavian Patrus, al I CUZA University, Romania Dr. Jam de Villiers, Fusion Studies, AEC, SO AFRICA Prof. M.A. Helloarg. Univarsity of Naral, SO AFRICA C.I.E.H.A.T., Fusion Div. Library, SPAIN Dr. Lennart Stenflo, Univarsity of UMEA, SHEDEN Library, Royal Institute of Taen, SWEDEN

Prof. Hans wilhelinson, Chalmars Univ of Tach, SWEDEN Contro Phys des Plasads. Ecole Polytech Fad, SwitzerLand Bibtiothenk, Fom-Inst Voor PIasan-Fysica, THE NETHERLANOS matin Durgut, Midole Eost Technicol University, TURKEY Dr. D.D. Ryutov, Sibarion Acad Sei, USSR Dr. G.A. Eliseer, Kurchotar institute, USSR Dr. V,A. Glukhikh, Inst Elactrophysical Apparatus, USSR Prof. O.S. Padichenko, Inst. of Phys. A Tech. USSR Dr. L.M. Kovrizhnykn, Institute of Gen. Physics, USSR Nuclear Res. Establishment, Julich Ltd., W. GERMANY Bibliothek, Inst. Fur Plasmoforschung, W. GERaMANY Dr. K. Schindler, Ruhr-Universitat Bochum, W. GERMANY ASDEX Reading Ra, cyo Wagner, IPP/Max-PIanck, W. GEPMANY Librarian, Maxtignck Institut, W. GERMANy

Prot. R.K. Janey, inst of Phys, Yugoslavia

\section{REPRODUCED FROM BEST REPRODUCED FROP: AVALLABLE COPY

